\title{
Barren Miocene granitoids in the Central Andean metallogenic belt, Chile: Geochemistry and Nd-Hf and U-Pb isotope systematics
}

\author{
Katja Deckart ${ }^{1}$, Estanislao Godoy ${ }^{2}$, Alfredo Bertens ${ }^{3}$, Daniela Jerez $^{4}$, Ayesha Saeed $^{5}$ \\ ${ }^{\prime}$ Departamento de Geología, Universidad de Chile, Plaza Ercilla 803, Casilla 13518, Santiago, Chile. \\ kdeckart@cec.uchile.cl \\ 2 V. Subercaseaux 4100, Pirque, Chile. \\ egodoyster@gmail.com \\ ${ }^{3}$ Department of Geology, Royal Holloway University of London, Egham, Surrey TW20 OEX, United Kingdom. \\ a.bertens@gl.rhul.ac.uk \\ ${ }^{4}$ Rosario Sur 91, Oficina 803, Las Condes, Santiago, Chile. \\ daniela_jerez@hotmail.com \\ ${ }_{5}^{5}$ ARC National Key Centre for Geochemical Evolution and Metallogeny of Continents (GEMOC), Department of Earth and Plane- \\ tary Sciences, Macquarie University Sydney, NSW 2109, Australia. \\ asaeed@els.mq.edu.au
}

\begin{abstract}
Four Middle-to-Late Miocene barren plutonic complexes that occur between the giant porphyry copper deposits of the central Chilean Andes were selected for U-Pb LA-ICPMS geochronology and Hf-isotope systematics on single zircon grains. Major and trace elements and Sr-Nd-Hf isotope whole rock geochemical studies were undertaken to compare with slightly younger or coeval barren and fertile intrusive rocks between $32^{\circ}$ and $34^{\circ} \mathrm{S}$. The studied granitoids yield resolvable crystallization ages of 11.3 $\pm 0.1 \mathrm{Ma}$ (Cerro Mesón Alto massif), 10.3 $\pm 0.2 \mathrm{Ma}$ (La Gloria pluton), $14.9 \pm 0.2 \mathrm{Ma} / 14.9 \pm 0.1 \mathrm{Ma}$ (Yerba Loca stock) and 11.2 $\pm 0.1 \mathrm{Ma} / 14.7 \pm 0.1 \mathrm{Ma}$ (San Francisco Batholith). Major and trace elements discard an adakitic signature as suggested for coeval porphyric intrusions at $32^{\circ} \mathrm{S}$, slightly younger mineralized porphyries at Río Blanco-Los Bronces deposit and other Cenozoic adakites. Volcanic host rocks are less fractionated than the intrusive rock units. The same observation can be made for the unmineralized northern plutons compared to the southern ones. Initial Sr-Nd isotope data show insignificant variation (0.703761-0.704118 and 0.5127580.512882 ), plotting in the mantle array. Trace element enrichment can be explained by addition of subducted-slab fluids and/or terrigenous sediments to the mantle wedge prior to and/or slight crustal input during magma ascent. Zircon grains separated from these barren intrusives share a similar initial $\varepsilon H f-d a t a$ variation for the younger age group (10-12 Ma; 7.04-9.54) and show a more scattered range for the older one (14-15 Ma; 8.50-15.34); both sets plot between the DM and CHUR evolution lines. There is evidence that magma evolution was slightly distinct through time from older to younger barren magmatism, compared to a few fertile porphyritic rocks from Río Blanco-Los Bronces porphyry copper deposit. It is suggested that chronological inconsistencies within these complexes might be related to differential shortening across the NE-SW-trending Yeso Valley transfer fault, assumed as coeval, which also explains the local lack of easterly magmatic arc front migration.
\end{abstract}


RESUMEN. Granitoides estériles del Mioceno en la franja metalogénica de los Andes Centrales, Chile: geoquímica e isotopía de Nd-Hf y U-Pb. Cuatro complejos plutónicos del Mioceno Temprano a Medio que se encuentran ubicados entre los depósitos de pórfidos gigantes de cobre de los Andes chileno centrales fueron seleccionados para un estudio de geocronología U-Pb por LA-ICPMS y de isótopos de Hf en granos individuales de circón. Estudios geoquímicos de elementos mayores y trazas y los isótopos de Sr-Nd-Hf en roca permiten compararlos con rocas intrusivas ligeramente más jóvenes o coetáneas estériles y fértiles entre $32^{\circ}$ y $34^{\circ} \mathrm{S}$. Los granitoides estudiados muestran edades de cristalización

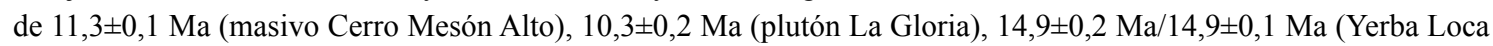
stock) y $11,2 \pm 0,1 \mathrm{Ma} / 14,7 \pm 0,1 \mathrm{Ma}$ (Batolito San Francisco). Elementos mayores y trazas excluyen una característica adakítica, como ha sido sugerido para los intrusivos porfídicos a los $32^{\circ} \mathrm{S}$, los pórfidos mineralizados ligeramente más jóvenes de Río Blanco-Los Bronces y otras adakitas del Cenozoico. Las rocas volcánicas de caja son menos fraccionadas que las unidades de rocas intrusivas. La misma conclusión se obtiene con relación a los plutones de la zona norte comparados a los del sur. Valores isotópico iniciales Sr-Nd muestran una variación insignificante (0,703761-0,704118 y 0,512758-0,512882) en el campo del manto. El enriquecimiento de elementos traza puede ser explicado a través de una adición de fluidos de la placa subducida y/o por sedimentos terrígenos transportados hacia la cuña de manto y/o por un leve aporte cortical durante el ascenso del magma. Granos de circón separados de los intrusivos estériles del grupo más joven (10-12 Ma; 7,04-9,54) comparten valores iniciales de $\varepsilon H f$, pero los intrusivos más antiguos (14-15 Ma; 8,50-15,34), en comparación, representan un rango más amplio; ambos rangos de valores se proyectan en la línea de evolución entre el DM y CHUR. Hay evidencia de que la evolución magmática fue ligeramente distinta a lo largo del tiempo desde un magmatismo joven y estéril hacia las rocas porfídicas mineralizadas de Río Blanco-Los Bronces. Se sugiere que la inconsistencia geocronológica entre los distintos complejos podría relacionarse con un acortamiento diferencial a través de una falla de transferencia NE-SO a lo largo del valle del ríoYeso, asumida coetánea, lo cual explicaría la falta, a nivel local, de migración del frente del arco magmático hacia el este.

Palabras clave: Magmatismo, Mioceno, Andes centrales, Sistemas isotópicos de Nd-Hfy U-Pb.

\section{Introduction}

Miocene-Pliocene magmatism in the Andean Cordillera of central Chile between latitudes $32^{\circ} \mathrm{S}$ and $34^{\circ} \mathrm{S}$ has been of major interest since it hosts world-class porphyry $\mathrm{Cu}(-\mathrm{Mo})$ deposits. Studies of magma source based on the $\mathrm{Sr}$ and $\mathrm{Nd}$ isotopic systematics of mineralized plutonic complexes have revealed the involvement of magmas having component signatures characteristic of mantle, subduction, lower and/or upper crust. Several authors (e.g., Stern, 1991; Stern and Puig, 1991; Skewes et al., 1991; Skewes and Stern, 1994; Kay and Mpodozis, 2001) have discussed the migration and petrogenetic evolution of the magmatism, suggesting that it was directly linked to the changing geometry of the subduction zone. The aim of these discussions was to develop a model for mineralization occurring in flat-slab areas of the Andean Cordillera. In contrast, the associated barren magmatism has generally not been considered, having been investigated separately by Kurtz et al. (1997), Kay et al. (1999) and Kay et al. (2005).
This paper adds geochronological, geochemical and isotopic data to the discussion of petrogenetic processes involved in the Miocene-Pliocene barren magmatism of the central Andean Cordillera. The new data set pretends to help and better understand the evolution of barren to fertile magmas related to subduction dynamics.

The geochronological and geochemical data presented here focus on four selected barren intrusive units of Miocene age, from south to north: Cerro Mesón Alto massif(CMA, $33^{\circ} 40^{\prime}$ 'S), La Gloria pluton (LGP,

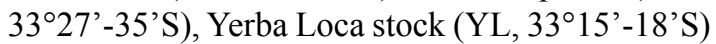
and San Francisco Batholith (SFB, $33^{\circ} 10^{\prime} \mathrm{S}$ ). They were emplaced between two supergiant Mio-Pliocene porphyry $\mathrm{Cu}$ (-Mo) deposits in the central Andes (Fig. 1). The first U-Pb and Hf-isotope LA-ICPMS data on single zircon grains and major and trace elements as well as $\mathrm{Sr}-\mathrm{Nd}-\mathrm{Hf}$ isotope data on whole rocks from these barren plutonic complexes presented here, allow to make progress on understanding the nature of the involved magma source(s) and their evolution in time. This new data set is compared with published analyses and interpretations for coeval, barren intrusions and slightly younger fertile porphyric rocks from the central Andean Cordillera. 


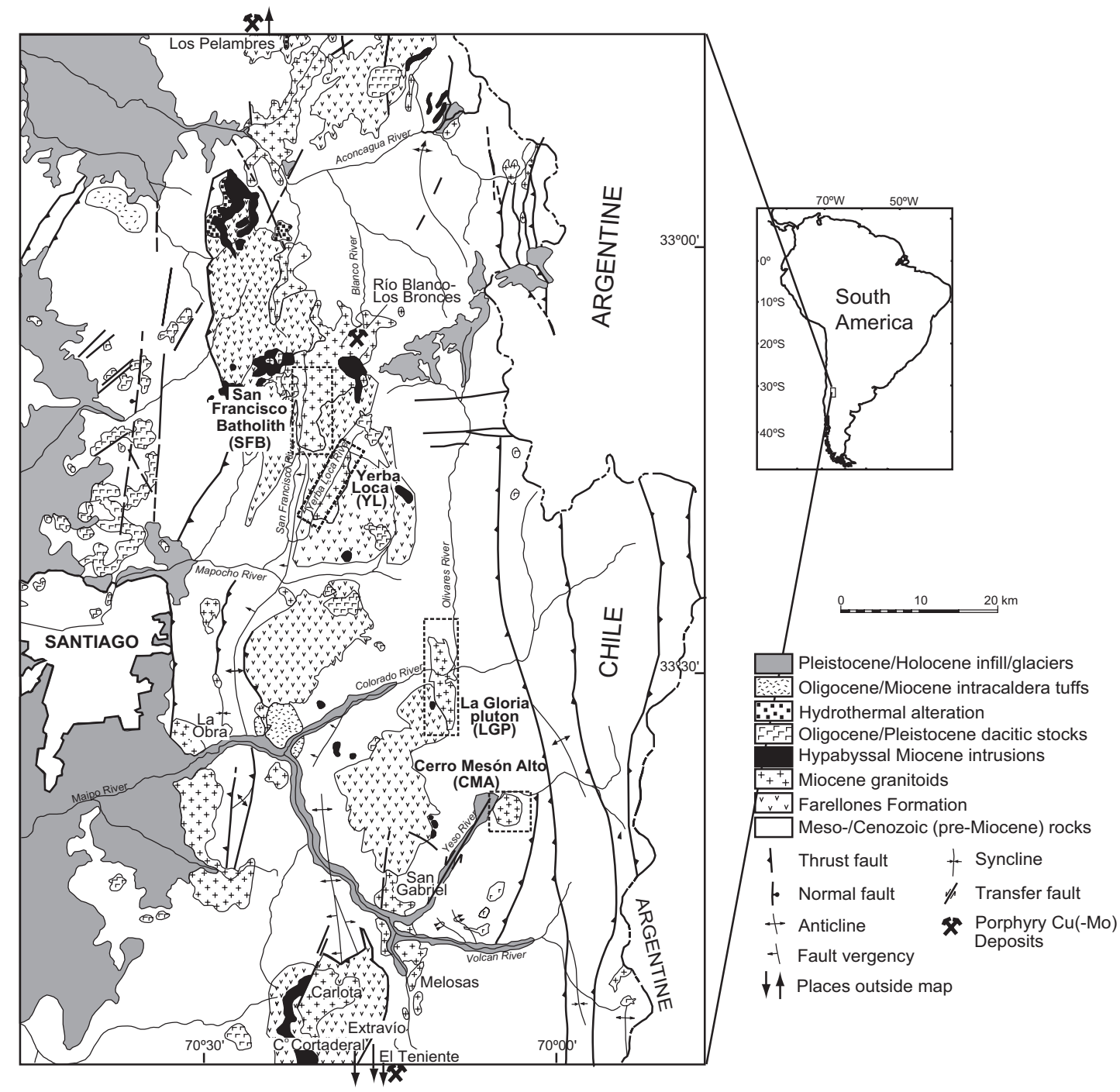

FIG. 1. Geological map with sample locations. Modified after Rivano et al. (1990).

Sample collection was undertaken in a limited region between $33^{\circ}$ and $34^{\circ} \mathrm{S}$, but the discussion addresses the entire Miocene-Pliocene metallogenic belt of the Main Andean Cordillera (Principal Cordillera; $32^{\circ}-34^{\circ} \mathrm{S}$ ) in central Chile.

\section{Plutonism at $33^{\circ}-34^{\circ} \mathrm{S}$ : current interpretation}

Miocene-Pliocene central Andean plutonic rocks are located at the southern limit and immediately south of the flat-slab segment $\left(\mathrm{ca} .29^{\circ}-34^{\circ} \mathrm{S}\right)$, which separates the central volcanic zone from the southern volcanic zone. This $c a .32^{\circ}-34^{\circ} \mathrm{S}-70^{\circ} 00^{\prime}-30^{\prime} \mathrm{W}$ belt hosts three major Miocene-Pliocene porphyry $\mathrm{Cu}(-\mathrm{Mo})$ deposits, from north to south, Los Pelambres, Río Blanco-Los Bronces and El Teniente (Fig. 1). Barren granitoids or smaller apophyses align with the three deposits. Mineral deposits along the Andean Cordillera may be linked to the convergence of the oceanic Nazca and South American continental plates (e.g., Sillitoe, 1974; Davidson and Mpodozis, 1991) and a causal relation between subduction and the nature (barren or fertile) of the associated magmatism has been proposed by e.g., Richards (2005).

In the study area, barren and mineralizationhosting granitoids of the San Francisco Batholith and the Teniente Plutonic Complex (cf. Kurtz et al., 1997; Kay et al., 2005), as well as smaller apophyses 
intrude either the Miocene Farellones Formation or the Eocene to earliest Miocene Abanico Formation. The generally assigned age of this plutonic magmatism is Middle Miocene (Table 1), but various unmineralized stocks lack precise geochronological information.

Of the studied plutonic units, the Cerro Mesón Alto massif (CMA), La Gloria pluton (LGP) and San Francisco Batholith (SFB) have been previously dated (Table 1), but not the Yerba Loca stock (YL). A single K-Ar age on biotite of 9.8 Ma (R.E. Drake, unpublished) has been presented for the La Gloria pluton by Cornejo and Mahood (1997) and interpreted as a cooling age, possibly close to its crystallization age. The Cerro Mesón
Alto granitoid, which represents the intrusive lower unit of the andesitic mountain peak, was dated by Kurtz et al. (1997), who named it after its outcrop south of 'Lago Yeso' rather than after its outcrop as a part of the Cerro Mesón Alto, and assigned it to the Teniente Plutonic Complex on the basis of a hornblende ${ }^{40} \mathrm{Ar} /{ }^{39} \mathrm{Ar}$ age of $12.4 \pm 2.5 \mathrm{Ma}$. Published $\mathrm{U}-\mathrm{Pb}$ ages on the San Francisco Batholith show a range of 8 to $12 \mathrm{Ma}$ (in Deckart et al., 2005); the younger zircon ages of 8-9 Ma were for samples from the Río Blanco-Los Bronces mine area and were interpreted as late, but still pre-mineralization (mineralization from ca. 6.3-4.3 Ma; Deckart et al., 2005), barren magmatic pulses of the San Francisco Batholith complex.

TABLE 1. PREVIOUS GEOCHRONOLOGICAL DATA OF MIO-PLIOCENE INTRUSIVES OF THE CENTRAL ANDES (33 ${ }^{\circ}-34^{\circ}$ S).

\begin{tabular}{|c|c|c|c|c|c|}
\hline Coordinates & Locality. Rock Type & Dated Material & Method & $\operatorname{Age}(M a) \pm 2 \sigma$ & References \\
\hline $32^{\circ} 55^{\prime}-70^{\circ} 20^{\prime}$ & Salto del Soldado. Granodiorite & Whole Rock & $\mathrm{K}-\mathrm{Ar}$ & $19.5 \pm 0.5$ & Vergara and Drake (1978) \\
\hline \multirow{4}{*}{$33^{\circ} 35^{\prime}-70^{\circ} 29^{\prime}$} & \multirow{4}{*}{ La Obra. Leucogranodiorite } & Hornblende & ${ }^{40} \mathrm{Ar} /{ }^{39} \mathrm{Ar}$ & $21.6 \pm 4.9$ & Kurtz et al. (1997) \\
\hline & & Biotite & ${ }^{40} \mathrm{Ar} /{ }^{39} \mathrm{Ar}$ & $19.6 \pm 0.5$ & Kurtz et al. (1997) \\
\hline & & Plagioclase & ${ }^{40} \mathrm{Ar} /{ }^{39} \mathrm{Ar}$ & $17.1 \pm 0.4$ & Kurtz et al. (1997) \\
\hline & & K-feldspar & ${ }^{40} \mathrm{Ar} /{ }^{39} \mathrm{Ar}$ & $16.2 \pm 0.3$ & Kurtz et al. (1997) \\
\hline \multirow{5}{*}{$33^{\circ} 47^{\prime}-70^{\circ} 13^{\prime}$} & \multirow{4}{*}{ San Gabriel. Granodiorite } & Biotite & $\mathrm{K}-\mathrm{Ar}$ & $13.9 \pm 0.8$ & Vergara and Drake (1978) \\
\hline & & Hornblende. Biotite & $\mathrm{K}-\mathrm{Ar}$ & $11.6 \pm 1.3$ & Vergara and Drake (1978) \\
\hline & & Plagioclase & $\mathrm{K}-\mathrm{Ar}$ & $10.6 \pm 0.3$ & Vergara and Drake (1978) \\
\hline & & Biotite & ${ }^{40} \mathrm{Ar} /{ }^{39} \mathrm{Ar}$ & $11.4 \pm 0.2$ & Kurtz et al. (1997) \\
\hline & \multicolumn{2}{|l|}{ El Teniente Plutonic Complex: } & & & \\
\hline $33^{\circ} 39^{\prime}-70^{\circ} 03^{\prime}$ & Lago Yeso & Hornblende & ${ }^{40} \mathrm{Ar} /{ }^{39} \mathrm{Ar}$ & $12.4 \pm 2.5$ & Kurtz et al. (1997) \\
\hline $33^{\circ} 54^{\prime}-70^{\circ} 20^{\prime}$ & La Carlota & Biotite & ${ }^{40} \mathrm{Ar} /{ }^{39} \mathrm{Ar}$ & $8.7 \pm 0.3$ & Kurtz et al. (1997) \\
\hline $34^{\circ} 05^{\prime}-70^{\circ} 07^{\prime}$ & Alfalfalito & Biotite & ${ }^{40} \mathrm{Ar} /{ }^{39} \mathrm{Ar}$ & $12.3 \pm 0.2$ & Kurtz et al. (1997) \\
\hline \multirow{4}{*}{$33^{\circ} 07-70^{\circ} 18^{\prime}$} & \multicolumn{2}{|l|}{ San Francisco Batholith: } & & & \\
\hline & Río Blanco Granodiorite & Zircon & $\mathrm{U}-\mathrm{Pb}$ & $11.96 \pm 0.4$ & Deckart et al. (2005) \\
\hline & Río Blanco Mine area. Granodiorite & Zircon & $\mathrm{U}-\mathrm{Pb}$ & $8.4 \pm 0.23$ & Deckart et al. (2005) \\
\hline & Río Blanco Mine area. Diorite & Zircon & $\mathrm{U}-\mathrm{Pb}$ & $8.83 \pm 0.05$ & Deckart et al. (2005) \\
\hline \multirow{4}{*}{$33^{\circ} 07-70^{\circ} 18^{\prime}$} & \multicolumn{2}{|l|}{ Late Porphyries: } & & & \\
\hline & Río Blanco Mine area. Porphyry & Zircon & $\mathrm{U}-\mathrm{Pb}$ & $6.32 \pm 0.09$ & Deckart et al. (2005) \\
\hline & Río Blanco Mine area. Porphyry & Zircon & $\mathrm{U}-\mathrm{Pb}$ & $5.84 \pm 0.04$ & Deckart et al. (2005) \\
\hline & Río Blanco Mine area. Porphyry & Zircon & $\mathrm{U}-\mathrm{Pb}$ & $5.23 \pm 0.07$ & Deckart et al. (2005) \\
\hline \multirow{2}{*}{$34^{\circ} 07-70^{\circ} 18^{\prime}$} & \multirow{2}{*}{ El Teniente Mine. Quartz-diorite to tonalite } & Zircon & $\mathrm{U}-\mathrm{Pb}$ & $6.11-6.46$ & Maksaev et al. (2004) \\
\hline & & Zircon & $\mathrm{U}-\mathrm{Pb}$ & $5.48-5.67$ & Maksaev et al. (2004) \\
\hline \multirow{3}{*}{$34^{\circ} 01-70^{\circ} 18^{\prime}$} & El Teniente Mine. Porphyries. Dacite & Zircon & $\mathrm{U}-\mathrm{Pb}$ & $5.28 \pm 0.10$ & Maksaev et al. (2004) \\
\hline & Latite Dyke & Zircon & $\mathrm{U}-\mathrm{Pb}$ & $4.82 \pm 0.09$ & Maksaev et al. (2004) \\
\hline & Post-ore dyke & Zircon & $\mathrm{U}-\mathrm{Pb}$ & $3.85 \pm 0.18$ & Maksaev et al. (2004) \\
\hline
\end{tabular}


Isotopic data indicate a decrease of ${ }^{87} \mathrm{Sr} /{ }^{86} \mathrm{Sr}$ and an increase of $\varepsilon \mathrm{Nd}$ isotopic signatures for Pleistoceneto-Holocene volcanic centers from the northern end of the Southern Volcanic Zone (NSVZ) $\left(33^{\circ} \mathrm{S}\right)$ to the south $\left(\mathrm{SSVZ},>36^{\circ} \mathrm{S}\right)$, coincident with crustal thinning from north to south (e.g., Kay et al., 2005). Regarding El Teniente and Río Blanco-Los Bronces $\mathrm{Cu}(-\mathrm{Mo})$ deposit, isotopic signature are increasing in ${ }^{87} \mathrm{Sr} /{ }^{86} \mathrm{Sr}$ but decreasing in $\varepsilon \mathrm{Nd}$ ratios with decreasing ages of magmatic rocks (Kay et al., 2005; Stern and Skewes, 1995). However, for the flat-slab segment north of the NSVZ, isotope studies demonstrated an increase of radiogenic ${ }^{87} \mathrm{Sr} /{ }^{86} \mathrm{Sr}$ signatures and a decrease of $\varepsilon \mathrm{Nd}$ values in west-to-east and north-tosouth directions, which coincide with the postulated younging of mineralization-hosted intrusive rocks in the same directions (e.g., Stern et al., 1984; Stern and Puig, 1991; Stern and Skewes, 1995; Skewes and Stern, 1996). This agrees with a southward younging of the mineralized centers in the central Cordillera between Los Pelambres and the coeval Río Blanco-Los Bronces and El Teniente $\mathrm{Cu}(-\mathrm{Mo})$ deposits (Deckart et al., 2005).

The geochemical patterns of the barren plutons have been compared with the contemporaneous, mainly volcanic, Farellones Formation and were interpreted to be possibly cogenetic (cf. LópezEscobar et al., 1979; Kay et al., 1995). In the more recent study by Kay et al. (2005) it is proposed that both magmatic trends might be linked to tectonic erosion, yet not to the inception of subduction of the Juan Fernández Ridge near $33^{\circ} \mathrm{S}$ at $10 \mathrm{Ma}$, as reported by Yáñez et al. (2001).

\section{Zircon $\mathrm{U}-\mathrm{Pb}$ ages and Hf-isotopes}

Three unmineralized granitoids and one intrusive, which belongs to the San Francisco Batholith complex, hosting the Río Blanco-Los Bronces porphyry copper deposit, were dated by the U-Pb LA-ICPMS method on single zircon grains. A summary of intrusion-related published ages of the area is presented in table 1. Analytical procedures are described in Appendix 1. U-Pb age data are documented in tables 2a-d. Hf-isotopic data for single zircon grains are summarized in tables $3 \mathrm{a}-\mathrm{d}$.

The Cerro Mesón Alto (CMA) massif was sampled south of the 'Lago Yeso' dam, about $90 \mathrm{~km}$ southeast of Santiago (Fig. 1). This massif intrudes the Eocene to earliest Miocene volcaniclastic Abanico Formation with sharp contacts. Twenty-five spot analyses on different zircon grains yield a mean crystallization age of $11.29 \pm 0.10$ Ma with a MSWD of 0.91 and a probability of 0.59 (FA-3; Figs. 2a, 2b; Table 2a). This age is slightly younger but within error of the one $\left(12.4 \pm 2.5 \mathrm{Ma} ;{ }^{40} \mathrm{Ar} /{ }^{39} \mathrm{Ar}\right)$ from Kurtz et al. (1997). These authors ascribed the low precision of the latter to the low potassium content and/or a slight excess of ${ }^{40} \mathrm{Ar}$, which is common in amphiboles and can easily lead to ages older than the 'true' crystallization age. Consequently, it is suggested that the $\mathrm{U}-\mathrm{Pb}$ zircon age of $11.29 \pm 0.10 \mathrm{Ma}$ obtained here is the best estimation of crystallization age of the Cerro Mesón Alto massif.

The La Gloria pluton (LGP) is located in the Andean foothills, $50 \mathrm{~km}$ east of Santiago. It also intrudes the volcaniclastic Abanico Formation, dated west of the pluton at 21.1 to $30.9 \mathrm{Ma}(\mathrm{Ar} / \mathrm{Ar}$ on plagioclase, Fock, 2005) (FA-4; Fig. 1; Table 2b). The weighted mean age calculated from nineteen ablations on different zircon grains is $10.34 \pm 0.15$ Ma with a MSWD of 0.46 and a probability of 0.97 (Figs. 2d, 2e). Combined with the above-mentioned 9.8 Ma K-Ar biotite cooling age this indicates a rapid cooling history for the La Gloria pluton.

The north-south orientated San Francisco Batholith was sampled at its southern border $c a .40 \mathrm{~km}$ northnortheast of Santiago. Published K-Ar and Ar/Ar ages of the San Francisco Batholith range between 18.5 Ma and 11.3 Ma (e.g., Warnaars et al., 1985; Deckart et al., 2005). A recently published zircon U-Pb IDTIMS age of $11.96 \pm 0.40 \mathrm{Ma}$ (Deckart et al., 2005) was interpreted as the crystallization age of an unmineralized rock unit close to the Río Blanco mine area, a few kilometres northeast of the localities sampled for this study. Two new U-Pb LA-ICPMS ages on single zircon grains from two distinct sites were obtained here. The first sample was taken from a locality adjacent to the mine site Los Bronces and gave a crystallization age of $11.16 \pm 0.07 \mathrm{Ma}$ (FA-7, Table 2c, Fig. 3a). The second was collected ca. $8 \mathrm{~km}$ to the southeast and yielded a crystallisation age of 14.74 $\pm 0.13 \mathrm{Ma}$ (FA-8, Table 2c, Figs. 3b).

The Yerba Loca (YL) stock is located $c a .30 \mathrm{~km}$ northeast of Santiago and close to the southeastern exposed limit of the San Francisco Batholith. In this as yet undated intrusive two U-Pb LA-ICPMS ages have been obtained. A total of eleven spots were used to calculate a crystallization age of $14.88 \pm 0.16 \mathrm{Ma}$ with an MSWD of 0.45 for sample FA- 9 and an age of $14.96 \pm 0.15 \mathrm{Ma}(\mathrm{n}=11)$ with a MSWD of 0.58 for sample FA-11 (Table 2d, Figs. 3c-d). Sample FA-11 

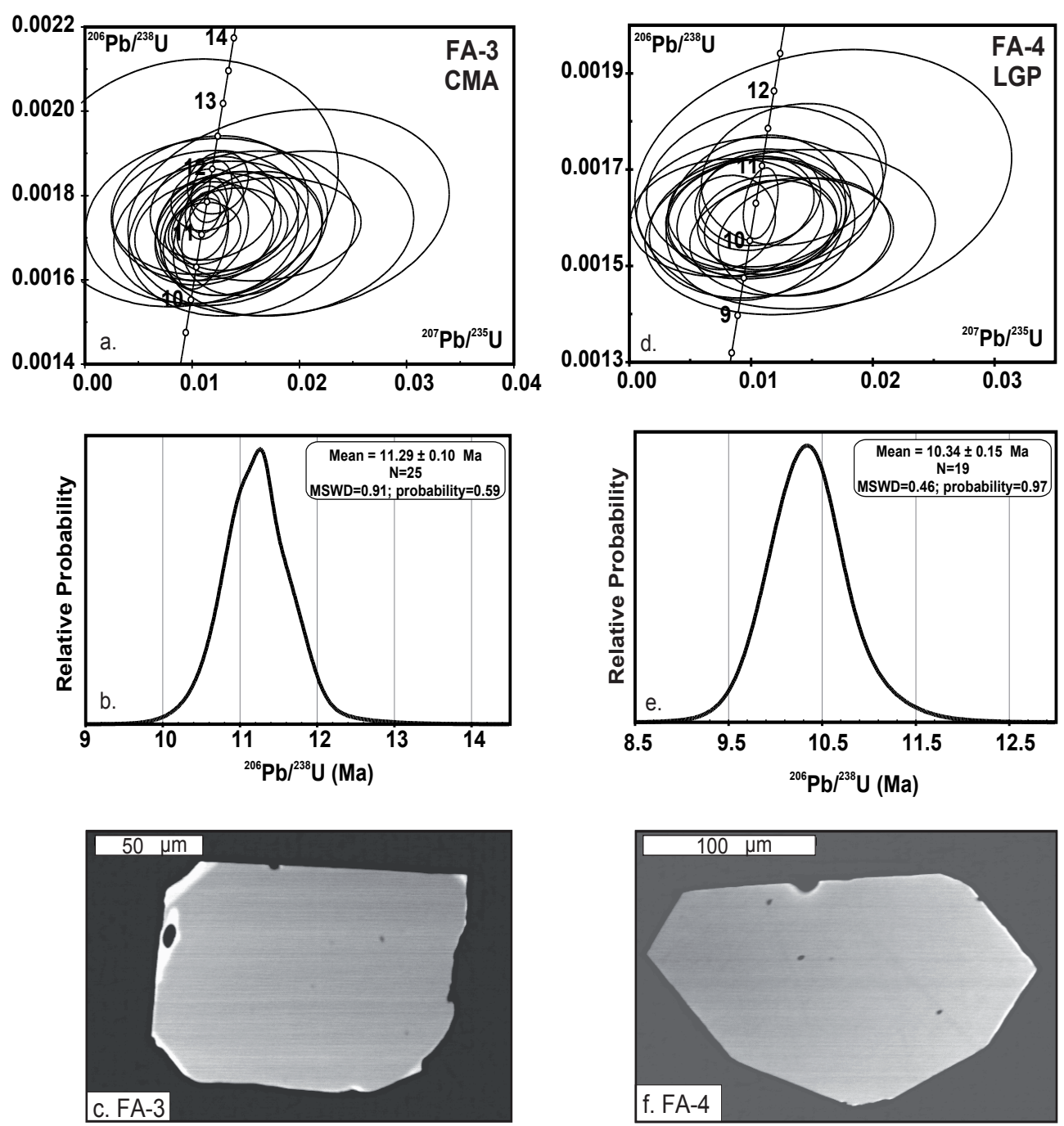

FIG. $2 .{ }^{206} \mathrm{~Pb} /{ }^{238} \mathrm{U}$ versus ${ }^{207} \mathrm{~Pb} /{ }^{235} \mathrm{U}$ relative probability plots for ${ }^{206} \mathrm{U} /{ }^{238} \mathrm{U}$ ages and BSE images of a representative zircon grain from (a-c) Cerro Mesón Alto massif (CMA; sample FA-3) and (d-f) La Gloria pluton (LGP; sample FA-4 (d-f)).

was collected $c a .20 \mathrm{~m}$ below and $100 \mathrm{~m}$ laterally from sample FA-9.

Thus the Cerro Mesón Alto massif has a slightly older age than the La Gloria pluton despite its location further east. On the other hand, only the younger age obtained from the San Francisco Batholith is coeval with the Cerro Mesón Alto massif U-Pb age. The Yerba Loca stock and most of the San Francisco Batholith are older than the Cerro Mesón Alto and La Gloria plutons. This pattern only partly confirms the postulated eastward younging of the magmatic arc (e.g., Stern and Puig, 1991; Skewes and Stern, 1996). However, because westward-vergent backfolding is restricted to the southern slopes of the Yeso
Valley, Godoy (2005) proposed a sinistral transfer fault along that valley, which could have been active during emplacement of the Cerro Mesón Alto massif (Fig. 1). The study area therefore shows a complex structural history with Late Miocene thrust faults trending mainly north-south, northeast-southwest and northwest-southeast, which might indicate differently displaced block structures in the metallogenic belt between $32-34^{\circ} \mathrm{S}$.

$\mathrm{Hf}$ isotopes for zircon minerals from all four plutonic complexes were also analyzed. Initial isotope ratios were calculated using the U-Pb zircon crystallization ages obtained during this study. The Cerro Mesón Alto massif and La Gloria pluton zircon samples 
TABLE 2. U-Pb LA-ICPMS AGES.

Ages [Ma] (common-Pb corrected)

Analysis No. $\quad{ }^{207} \mathrm{~Pb} /{ }^{206} \mathrm{~Pb} \quad \pm 2 \sigma \quad{ }^{207} \mathrm{~Pb} /{ }^{235} \mathrm{U} \quad \pm 2 \sigma \quad{ }^{206} \mathrm{~Pb} /{ }^{238} \mathrm{U} \quad \quad \pm 2 \sigma \quad{ }^{208} \mathrm{~Pb} /{ }^{232} \mathrm{Th} \quad \pm 2 \sigma \quad \mathrm{RHO}^{2} \quad$

2a. U-Pb LA-ICPMS ages for sample FA-3, Cerro Mesón Alto.

\begin{tabular}{|c|c|c|c|c|c|c|c|c|c|}
\hline FA-3-01 & 307 & 1,022 & 13 & 8 & 11 & 1 & 15 & 4 & 0.128 \\
\hline FA-3-02 & 1,209 & 922 & 19 & 10 & 11 & 1 & 15 & 6 & 0.186 \\
\hline FA-3-03 & 140 & 644 & 12 & 4 & 11.4 & 0.6 & 11 & 2 & 0.142 \\
\hline FA-3-04 & 3 & 422 & 11 & 2 & 11 & 0.6 & 11 & 6 & 0.303 \\
\hline FA-3-05 & 1,154 & 1,172 & 19 & 12 & 11.3 & 1.2 & 11 & 6 & 0.176 \\
\hline FA-3-06 & 1 & 578 & 11 & 4 & 11.1 & 0.6 & 11 & 2 & 0.180 \\
\hline FA-3-07 & 812 & 626 & 16 & 6 & 11 & 0.8 & 12 & 2 & 0.204 \\
\hline FA-3-08 & 679 & 1,030 & 15 & 8 & 11 & 1 & 10 & 4 & 0.159 \\
\hline FA-3-09 & 528 & 788 & 14 & 6 & 11 & 0.8 & 11 & 2 & 0.165 \\
\hline FA-3-10 & 486 & 644 & 14 & 4 & 11.1 & 0.6 & 14 & 2 & 0.142 \\
\hline FA-3-11 & 70 & 228 & 11.9 & 1.6 & 11.7 & 0.4 & 12.1 & 0.8 & 0.251 \\
\hline FA-3-12 & 88 & 958 & 12 & 8 & 11.4 & 1 & 9 & 4 & 0.123 \\
\hline FA-3-13 & 27 & 930 & 11 & 8 & 11.3 & 0.8 & 12 & 2 & 0.109 \\
\hline FA-3-14 & 497 & 722 & 14 & 6 & 11 & 0.8 & 12 & 2 & 0.180 \\
\hline FA-3-15 & 302 & 674 & 13 & 4 & 11.1 & 0.6 & 12 & 2 & 0.153 \\
\hline FA-3-16 & 56 & 728 & 11 & 6 & 11 & 0.6 & 10.5 & 1.6 & 0.123 \\
\hline FA-3-17 & 29 & 466 & 11 & 2 & 11 & 0.4 & 11.1 & 0.8 & 0.134 \\
\hline FA-3-18 & 9 & 178 & 11.3 & 1.2 & 11.3 & 0.2 & 11.3 & 0.4 & 0.220 \\
\hline FA-3-19 & 10 & 432 & 12 & 2 & 11.5 & 0.4 & 12.3 & 1.2 & 0.139 \\
\hline FA-3-20 & -446 & 1,684 & 10 & 12 & 11.8 & 1.6 & 18 & 4 & 0.107 \\
\hline FA-3-21 & 944 & 966 & 17 & 8 & 11.1 & 0.6 & 10.6 & 0.8 & 0.133 \\
\hline FA-3-22 & 68 & 1,208 & 11 & 10 & 11.2 & 1 & 7 & 4 & 0.113 \\
\hline FA-3-23 & 12 & 320 & 11.1 & 2 & 11.1 & 0.4 & 11.7 & 0.8 & 0.199 \\
\hline FA-3-24 & 24 & 244 & 11.4 & 1.6 & 11.4 & 0.4 & 10.9 & 0.8 & 0.240 \\
\hline FA-3-25 & 151 & 358 & 13 & 2 & 11.8 & 0.4 & 11.7 & 1.2 & 0.175 \\
\hline
\end{tabular}

2b. U-Pb LA-ICPMS ages for sample FA-4, La Gloria pluton.

\begin{tabular}{|c|c|c|c|c|c|c|c|c|c|}
\hline FA-4-01 & 133 & 1,078 & 11 & 8 & 10.7 & 1 & 7 & 4 & 0.118 \\
\hline FA-4-02 & -7 & 604 & 10 & 4 & 10.5 & 0.6 & 9.3 & 1.6 & 0.126 \\
\hline FA-4-03 & 98 & 172 & 10.9 & 1.2 & 10.5 & 0.4 & 11 & 4 & 0.342 \\
\hline FA-4-04 & 76 & 616 & 11 & 4 & 10.6 & 0.6 & 10.9 & 1.6 & 0.128 \\
\hline FA-4-05 & 832 & 774 & 14 & 6 & 10 & 0.6 & 9 & 4 & 0.159 \\
\hline FA-4-06 & 676 & 948 & 13 & 6 & 10 & 0.6 & 13 & 4 & 0.125 \\
\hline FA-4-08 & 63 & 868 & 11 & 6 & 10.3 & 0.6 & 8 & 4 & 0.107 \\
\hline FA-4-09 & 344 & 700 & 12 & 4 & 10.2 & 0.6 & 11 & 2 & 0.132 \\
\hline FA-4-10 & -25 & 556 & 8 & 4 & 10.2 & 0.6 & 10.3 & 1.2 & 0.121 \\
\hline FA-4-11 & 21 & 1,146 & 10 & 8 & 10.4 & 0.8 & 9 & 4 & 0.096 \\
\hline FA-4-12 & 533 & 830 & 14 & 6 & 10.9 & 0.8 & 11 & 4 & 0.155 \\
\hline FA-4-13 & 13 & 998 & 10 & 6 & 10.2 & 0.8 & 11 & 4 & 0.111 \\
\hline FA-4-14 & 634 & 1,288 & 13 & 10 & 10.1 & 1 & 11 & 4 & 0.121 \\
\hline FA-4-15 & 274 & 714 & 11 & 4 & 10.2 & 0.6 & 13 & 2 & 0.122 \\
\hline FA-4-16 & 50 & 834 & 11 & 6 & 10.3 & 1 & 14 & 2 & 0.151 \\
\hline FA-4-17 & 898 & 1,398 & 16 & 12 & 10.8 & 1.4 & 14 & 6 & 0.165 \\
\hline FA-4-18 & 89 & 874 & 11 & 6 & 10.4 & 0.6 & 9 & 4 & 0.107 \\
\hline FA-4-19 & 35 & 836 & 10 & 6 & 10.3 & 0.6 & 10 & 2 & 0.111 \\
\hline FA-4-20 & -22 & 1,080 & 8 & 6 & 10.3 & 0.6 & 8 & 4 & 0.077 \\
\hline
\end{tabular}


Table 2. continued.

Ages [Ma] (common-Pb corrected)

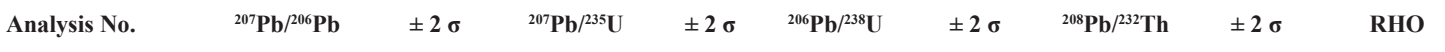

2c. U-Pb zircon LA-ICPMS data for FA-7 and FA-8, San Francisco Batholith.

\begin{tabular}{|c|c|c|c|c|c|c|c|c|c|}
\hline FA-7-01 & 164 & 226 & 11.8 & 1.6 & 11.1 & 0.6 & 12 & 4 & 0.339 \\
\hline FA-7-02 & 137 & 700 & 12 & 6 & 11.3 & 1.2 & 14 & 6 & 0.216 \\
\hline FA-7-03 & 39 & 132 & 11.2 & 1 & 11.1 & 0.2 & 11.1 & 0.8 & 0.286 \\
\hline FA-7-04 & 45 & 60 & 11.2 & 0.4 & 11.1 & 0.2 & 10.3 & 0.4 & 0.540 \\
\hline FA-7-05 & 311 & 738 & 13 & 6 & 11.1 & 1.2 & 10 & 2 & 0.223 \\
\hline FA-7-06 & 772 & 174 & 15.4 & 1.8 & 11 & 0.6 & 15 & 1.6 & 0.390 \\
\hline FA-7-07 & 110 & 106 & 11.5 & 0.8 & 11 & 0.2 & 10.7 & 1.6 & 0.369 \\
\hline FA-7-08 & 85 & 104 & 11.5 & 0.8 & 11.2 & 0.2 & 11.9 & 0.8 & 0.365 \\
\hline FA-7-09 & 22 & 322 & 11.3 & 2 & 11.3 & 0.4 & 10.5 & 1.2 & 0.196 \\
\hline FA-7-10 & 93 & 516 & 12 & 4 & 11.7 & 0.8 & 11.7 & 1.6 & 0.208 \\
\hline FA-7-11 & 144 & 442 & 12 & 4 & 11.6 & 0.8 & 12 & 2 & 0.261 \\
\hline FA-7-12 & 69 & 892 & 12 & 8 & 11.3 & 1.4 & 11 & 4 & 0.193 \\
\hline FA-7-13 & 183 & 238 & 12.1 & 1.6 & 11.2 & 0.4 & 10 & 2 & 0.261 \\
\hline FA-7-15 & 321 & 346 & 13 & 2 & 11.3 & 0.6 & 11.5 & 1.6 & 0.285 \\
\hline FA-7-17 & 18 & 82 & 11.3 & 0.6 & 11.3 & 0.2 & 10.5 & 0.4 & 0.387 \\
\hline FA-7-18 & 11 & 166 & 11 & 1 & 11 & 0.2 & 10.7 & 0.4 & 0.237 \\
\hline FA-7-19 & 2,997 & 182 & 55 & 12 & 11.8 & 1.6 & 25 & 6 & 0.617 \\
\hline FA-7-20 & 1,052 & 1,316 & 18 & 14 & 11 & 2 & 11 & 6 & 0.246 \\
\hline FA-7-21 & 13 & 192 & 11.6 & 1.4 & 11.5 & 0.4 & 14.7 & 1.2 & 0.282 \\
\hline FA-7-22 & 122 & 154 & 11.7 & 1 & 11.2 & 0.2 & 10.9 & 0.4 & 0.267 \\
\hline FA-8-01 & 56 & 688 & 15 & 6 & 14.8 & 1.2 & 17 & 4 & 0.169 \\
\hline FA-8-02 & 58 & 270 & 15 & 2 & 14.9 & 0.6 & 15.4 & 1.6 & 0.269 \\
\hline FA-8-03 & 21 & 338 & 15 & 2 & 14.9 & 0.6 & 15.2 & 1.6 & 0.227 \\
\hline FA-8-04 & 128 & 240 & 16 & 2 & 14.9 & 0.6 & 14.7 & 1.2 & 0.253 \\
\hline FA-8-05 & 67 & 274 & 15 & 2 & 14.4 & 0.6 & 14 & 2 & 0.231 \\
\hline FA-8-06 & 1,530 & 584 & 30 & 12 & 14.7 & 1.6 & 22 & 6 & 0.292 \\
\hline FA-8-07 & 59 & 192 & 15.2 & 1.8 & 14.9 & 0.6 & 14.7 & 1.6 & 0.347 \\
\hline FA- $8-08$ & 26 & 344 & 15 & 2 & 14.6 & 0.6 & 14.5 & 1.2 & 0.190 \\
\hline FA-8-09 & 675 & 260 & 20 & 4 & 14.9 & 0.6 & 26 & 4 & 0.278 \\
\hline FA-8-10 & 98 & 66 & 15.4 & 0.8 & 14.9 & 0.4 & 14.5 & 0.8 & 0.536 \\
\hline FA-8-11 & 27 & 128 & 15 & 1.2 & 15 & 0.4 & 14.3 & 0.8 & 0.310 \\
\hline FA-8-12 & 94 & 290 & 15 & 2 & 14.8 & 0.6 & 14.7 & 1.2 & 0.217 \\
\hline FA-8-13 & 510 & 422 & 19 & 4 & 15 & 1 & 15 & 2 & 0.253 \\
\hline FA-8-14 & 44 & 456 & 15 & 4 & 14.9 & 0.6 & 17 & 2 & 0.166 \\
\hline FA-8-15 & 44 & 284 & 15 & 2 & 14.9 & 0.4 & 13.5 & 1.2 & 0.171 \\
\hline FA-8-16 & 121 & 346 & 16 & 2 & 14.9 & 0.6 & 12.5 & 1.6 & 0.225 \\
\hline FA-8-18 & 221 & 236 & 16.3 & 2 & 14.9 & 0.4 & 14.5 & 0.8 & 0.209 \\
\hline FA-8-19 & 51 & 300 & 15 & 2 & 14.6 & 0.4 & 15 & 1.2 & 0.166 \\
\hline FA- $8-20$ & 64 & 328 & 16 & 2 & 15.5 & 0.6 & 16.2 & 1.6 & 0.226 \\
\hline FA-8-21 & 14 & 46 & 14.4 & 0.6 & 14.4 & 0.2 & 14.3 & 0.4 & 0.511 \\
\hline
\end{tabular}


Table 2. continued.

\begin{tabular}{|c|c|c|c|c|c|c|c|c|c|}
\hline \multicolumn{10}{|c|}{ Ages [Ma] (common-Pb corrected) } \\
\hline Analysis No. & ${ }^{207} \mathrm{~Pb} /{ }^{206} \mathrm{~Pb}$ & $\pm 2 \sigma$ & ${ }^{207} \mathbf{P b} \mathbf{b}^{235} \mathbf{U}$ & $\pm 2 \sigma$ & ${ }^{206} \mathbf{P b} \mathbf{b}^{238} \mathbf{U}$ & $\pm 2 \sigma$ & ${ }^{208} \mathbf{P b} /{ }^{232} \mathbf{T h}$ & $\pm 2 \sigma$ & RHO \\
\hline \multicolumn{10}{|c|}{ 2d. U-Pb zircon LA-ICPMS data for FA-9 and FA-11, Yerba Loca. } \\
\hline FA-9-01 & 15 & 350 & 15 & 2 & 15.1 & 0.4 & 15.4 & 1.2 & 0.147 \\
\hline FA-9-02 & 19 & 1,090 & 15 & 10 & 15.1 & 1 & 21 & 4 & 0.106 \\
\hline FA-9-03 & 22 & 382 & 15 & 4 & 14.9 & 0.6 & 15.4 & 1.6 & 0.162 \\
\hline FA-9-04 & 20 & 290 & 15 & 2 & 15 & 0.4 & 14.5 & 1.2 & 0.164 \\
\hline FA-9-05 & 15 & 252 & 15 & 2 & 15 & 0.4 & 15 & 1.2 & 0.168 \\
\hline FA-9-06 & 23 & 316 & 15 & 4 & 14.7 & 0.6 & 14.3 & 1.6 & 0.204 \\
\hline FA-9-07 & 332 & 476 & 17 & 4 & 14.7 & 0.6 & 14 & 2 & 0.181 \\
\hline FA-9-08 & -23 & 254 & 14.5 & 2 & 14.7 & 0.4 & 15.8 & 1.2 & 0.199 \\
\hline FA-9-09 & 40 & 486 & 15 & 4 & 14.7 & 0.6 & 15 & 2 & 0.115 \\
\hline FA-9-10 & 18 & 550 & 15 & 4 & 14.6 & 0.8 & 16 & 4 & 0.158 \\
\hline FA-9-15 & 25 & 644 & 15 & 6 & 14.9 & 1 & 14 & 2 & 0.136 \\
\hline FA-11-01 & 18 & 158 & 15 & 1.6 & 15 & 0.4 & 15.4 & 1.2 & 0.255 \\
\hline FA-11-02 & 15 & 312 & 15 & 2 & 14.9 & 0.6 & 15 & 1.6 & 0.245 \\
\hline FA-11-03 & 34 & 160 & 15.3 & 1.8 & 15.3 & 0.6 & 15.4 & 1.2 & 0.282 \\
\hline FA-11-05 & 16 & 246 & 15 & 2 & 15 & 0.6 & 14.5 & 1.6 & 0.278 \\
\hline FA-11-06 & 39 & 258 & 15 & 2 & 14.6 & 0.6 & 15.4 & 1.6 & 0.241 \\
\hline FA-11-08 & 16 & 190 & 15.2 & 1.8 & 15.1 & 0.4 & 15.8 & 0.8 & 0.213 \\
\hline FA-11-09 & 47 & 170 & 15 & 1.8 & 14.8 & 0.6 & 14.1 & 1.6 & 0.301 \\
\hline FA-11-11 & 31 & 202 & 15.3 & 1.8 & 15.2 & 0.6 & 16 & 1.6 & 0.274 \\
\hline FA-11-13 & 14 & 314 & 15 & 2 & 14.6 & 0.6 & 15 & 1.2 & 0.210 \\
\hline FA-11-14 & 25 & 184 & 15 & 1.8 & 14.9 & 0.4 & 15 & 1.2 & 0.227 \\
\hline FA-11-16 & 36 & 534 & 15 & 6 & 14.6 & 0.8 & 15 & 2 & 0.146 \\
\hline FA-11-21 & 31 & 200 & 15.1 & 1.8 & 15 & 0.4 & 15.4 & 1.6 & 0.208 \\
\hline
\end{tabular}

$\mathrm{RHO}=\mathrm{RSD}{ }^{206} \mathrm{~Pb} /{ }^{238} \mathrm{U}$ versus $\mathrm{RSD}{ }^{207} \mathrm{~Pb} /{ }^{235} \mathrm{U}$.

show initial $\varepsilon \mathrm{Hf}$ values ranging from +7.04 to +9.60 and from +7.13 to +8.93 for FA-3 and FA-4, respectively. The younger San Francisco Batholith zircon sample (FA-7) yields similar $\varepsilon \mathrm{Hf}_{\mathrm{i}}$ values from +7.04 to +8.84 . In contrast, the older zircon sample FA- 8 ranges between $\varepsilon \mathrm{Hf}_{\mathrm{i}}+8.50$ and $\varepsilon \mathrm{Hf}_{\mathrm{i}}+12.38$, which is slightly less radiogenic than the coeval zircon samples from the Yerba Loca stock $\left[\varepsilon \mathrm{Hf}_{\mathrm{i}}=+9.20\right.$ to +14.49 (FA-9) and +11.39 to +15.34 (FA-11)].

Calculated $\mathrm{Hf}$ model ages $\left(\mathrm{Hf}-\mathrm{T}_{\mathrm{DM}}\right)$, which are interpreted in the same way as $\mathrm{Nd}$ model ages, range from 300 to 390 Ma for sample FA-3 from the Cerro Mesón Alto massif and between 320 and $380 \mathrm{Ma}$ for sample FA-4 from the La Gloria pluton. The younger
San Francisco Batholith zircon sample (FA-7) shows a similar $\mathrm{T}_{\mathrm{DM}}$ age range from 320 to $390 \mathrm{Ma}$. The older rock sample from the San Francisco Batholith (FA-8) ranges in $\mathrm{T}_{\mathrm{DM}}$ ages from $194 \mathrm{Ma}$ to $340 \mathrm{Ma}$ whereas the Yerba Loca stock zircons (FA-9, FA-11) indicate the widest $\mathrm{T}_{\mathrm{DM}}$ age range between $76 \mathrm{Ma}$ and 310 $\mathrm{Ma}$, pointing to an inhomogeneous source of more juvenile mantle residuals. If a direct separation of these magmas from depleted asthenospheric mantle is assumed, initial epsilon Hf- values should be all greater than +10 . Since the values range generally around +10 , the magmas most probably were not derived directly from a depleted MORB-like mantle source. 

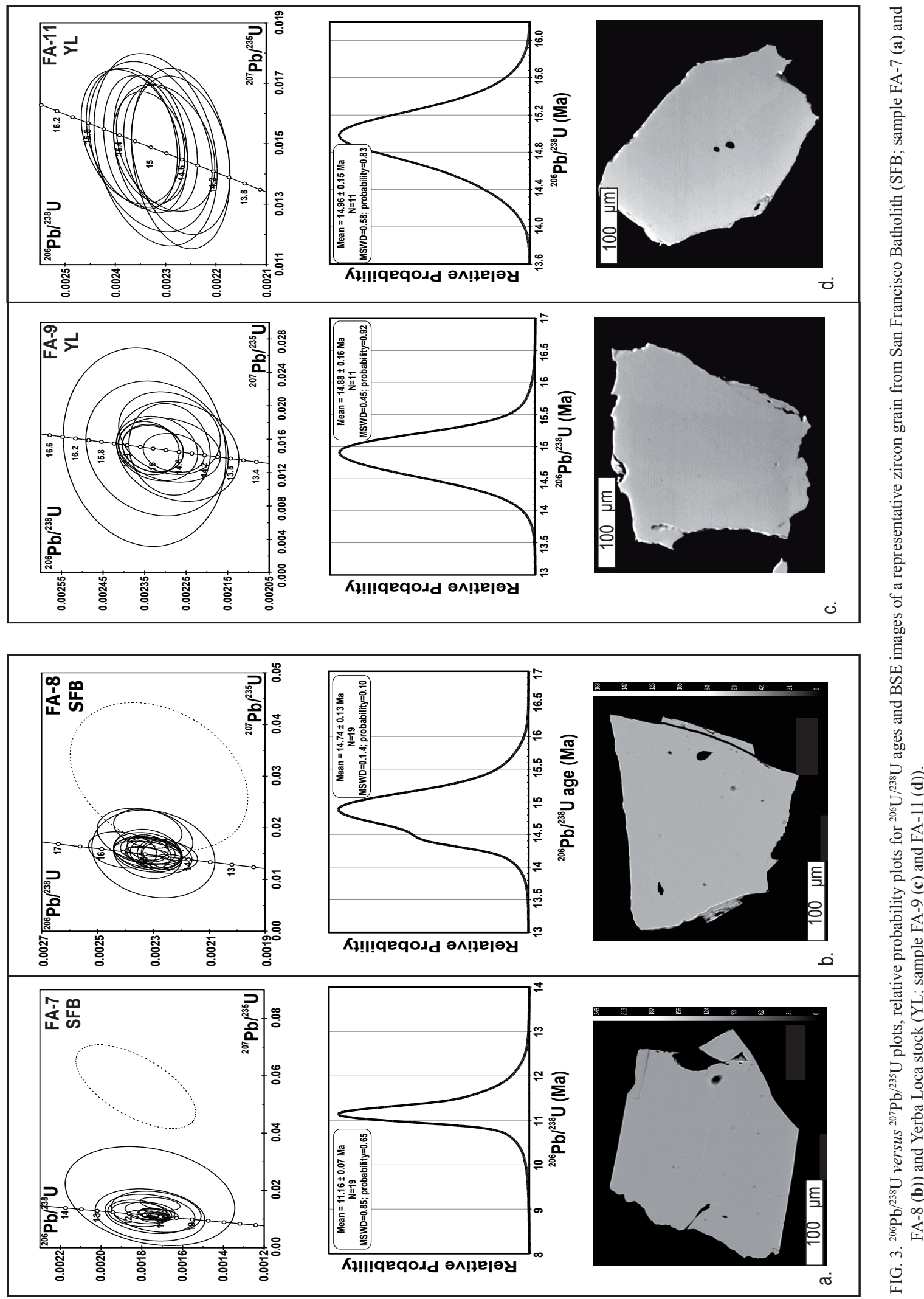


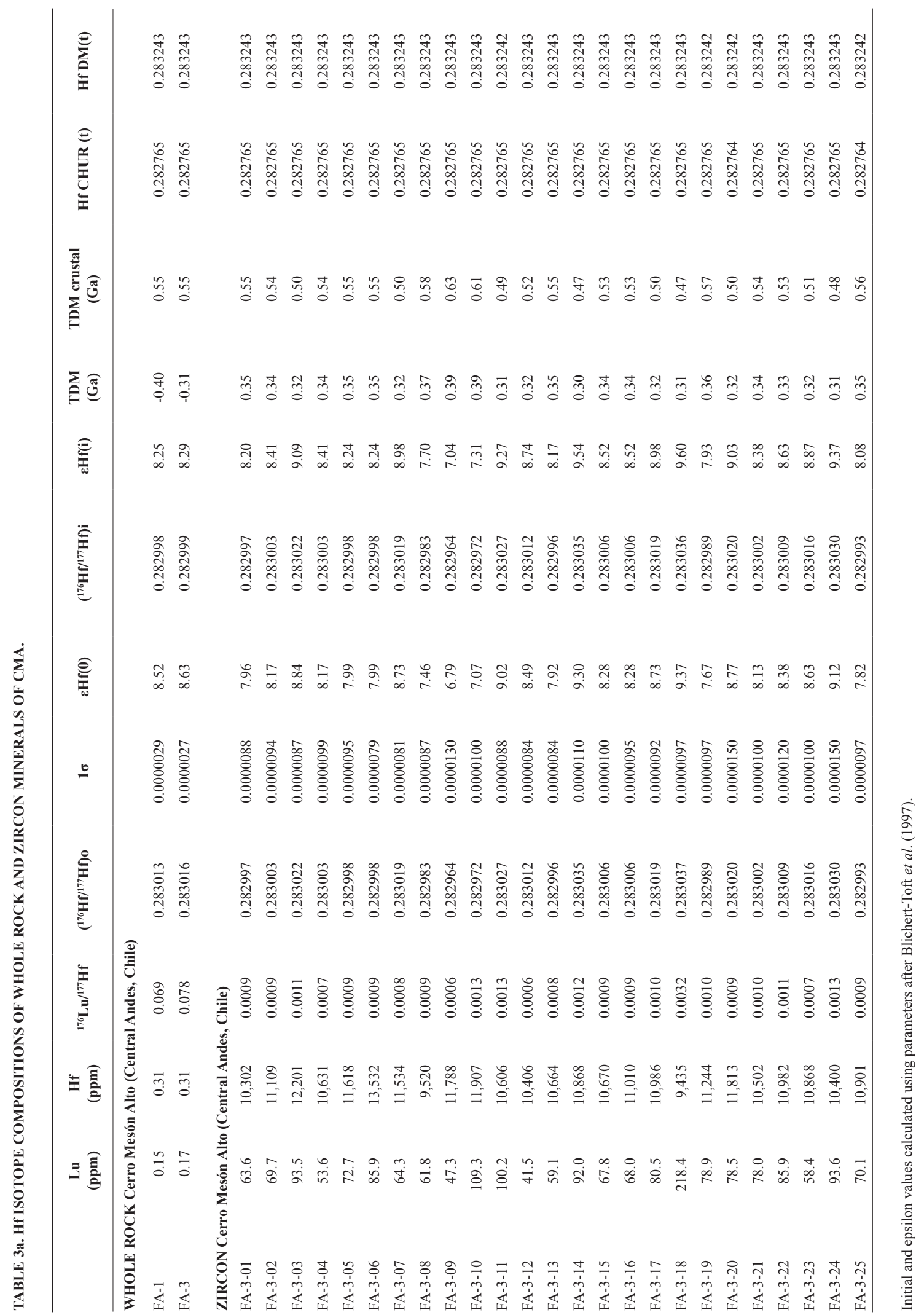




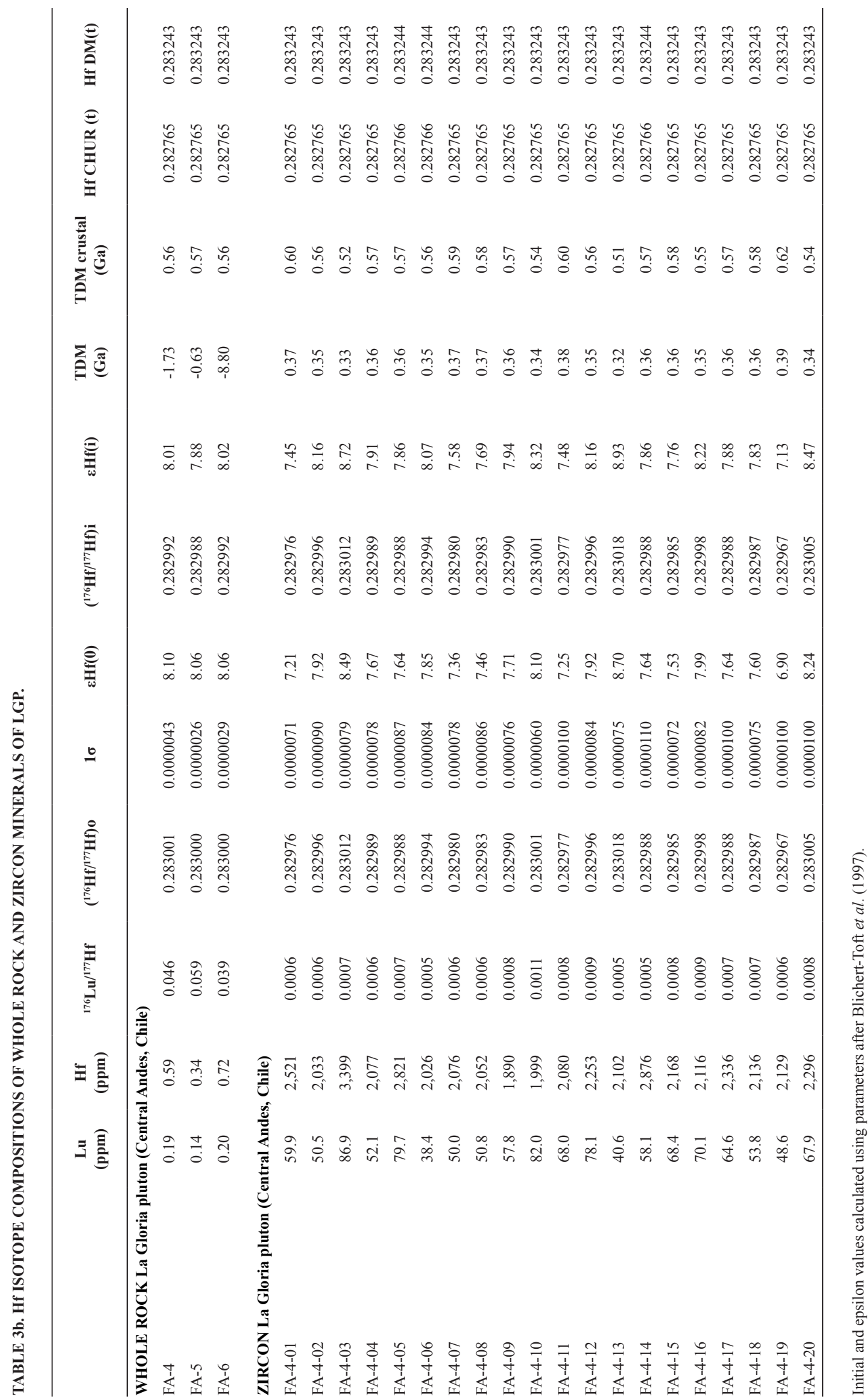


TABLE 3c. Hf ISOTOPE COMPOSITIONS OF WHOLE ROCK AND ZIRCON MINERALS OF SAN FRANCISCO BATHOLITH.

\begin{tabular}{|c|c|c|c|c|c|c|c|c|c|c|c|c|}
\hline & $\begin{array}{c}\text { Lu } \\
(\mathbf{p p m})\end{array}$ & $\begin{array}{c}\text { Hf } \\
(\mathbf{p p m})\end{array}$ & ${ }^{176} \mathbf{L u} /{ }^{177} \mathbf{H f}$ & $\left({ }^{176} \mathbf{H f} /{ }^{177} \mathbf{H f}\right) \mathbf{o}$ & $1 \sigma$ & $\varepsilon H f(0)$ & $\left({ }^{176} \mathbf{H f} /{ }^{177} \mathrm{Hf}\right) \mathrm{i}$ & $\varepsilon \mathbf{H f}(\mathbf{i})$ & $\begin{array}{l}\text { TDM } \\
\text { (Ga) }\end{array}$ & $\begin{array}{c}\text { TDM } \\
\text { crustal } \\
\text { (Ga) }\end{array}$ & Hf CHUR (t) & Hf DM(t \\
\hline \multicolumn{13}{|c|}{ WHOLE ROCK San Francisco Batholith (Central Andes, Chile) } \\
\hline FA-7 & 0.19 & 5.39 & 0.00501 & 0.283010 & 0.000003 & 8.42 & 0.283009 & 8.63 & 0.37 & 0.52 & 0.282762 & 0.283240 \\
\hline FA-8 & 0.29 & 5.33 & 0.00773 & 0.283010 & 0.000010 & 8.42 & 0.283008 & 8.67 & 0.40 & 0.53 & 0.282762 & 0.283240 \\
\hline \multicolumn{13}{|c|}{ ZIRCON San Francisco Batholith (Central Andes, Chile) } \\
\hline FA-7-01 & 62 & 14,798 & 0.00059 & 0.282964 & 0.000006 & 6.79 & 0.282964 & 7.04 & 0.39 & 0.62 & 0.282765 & 0.283243 \\
\hline FA-7-02 & 120 & 16,556 & 0.00101 & 0.283015 & 0.000009 & 8.59 & 0.283015 & 8.84 & 0.32 & 0.51 & 0.282765 & 0.283243 \\
\hline FA-7-03 & 82 & 15,161 & 0.00075 & 0.282997 & 0.000008 & 7.96 & 0.282997 & 8.20 & 0.35 & 0.55 & 0.282765 & 0.283243 \\
\hline FA-7-04 & 59 & 16,024 & 0.00051 & 0.282987 & 0.000006 & 7.60 & 0.282987 & 7.85 & 0.36 & 0.57 & 0.282765 & 0.283243 \\
\hline FA-7-05 & 52 & 9,755 & 0.00074 & 0.282993 & 0.000008 & 7.82 & 0.282993 & 8.06 & 0.35 & 0.56 & 0.282765 & 0.283243 \\
\hline FA-7-06 & 72 & 10,444 & 0.00096 & 0.282967 & 0.000009 & 6.90 & 0.282967 & 7.14 & 0.39 & 0.62 & 0.282765 & 0.283243 \\
\hline FA-7-07 & 102 & 14,943 & 0.00095 & 0.282991 & 0.000006 & 7.74 & 0.282991 & 7.99 & 0.36 & 0.57 & 0.282765 & 0.283243 \\
\hline FA-7-08 & 52 & 12,331 & 0.00058 & 0.282967 & 0.000006 & 6.90 & 0.282967 & 7.15 & 0.39 & 0.62 & 0.282765 & 0.283243 \\
\hline FA-7-09 & 86 & 10,721 & 0.00112 & 0.283005 & 0.000008 & 8.24 & 0.283005 & 8.49 & 0.34 & 0.54 & 0.282765 & 0.283243 \\
\hline FA-7-10 & 87 & 13,752 & 0.00088 & 0.283000 & 0.000006 & 8.06 & 0.283000 & 8.32 & 0.34 & 0.55 & 0.282765 & 0.283242 \\
\hline FA-7-11 & 93 & 12,083 & 0.00107 & 0.283000 & 0.000007 & 8.06 & 0.283000 & 8.32 & 0.35 & 0.55 & 0.282765 & 0.283242 \\
\hline FA-7-12 & 121 & 10,315 & 0.00163 & 0.282990 & 0.000010 & 7.71 & 0.282990 & 7.95 & 0.37 & 0.57 & 0.282765 & 0.283243 \\
\hline FA-7-13 & 50 & 11,318 & 0.00061 & 0.283000 & 0.000009 & 8.06 & 0.283000 & 8.31 & 0.34 & 0.55 & 0.282765 & 0.283243 \\
\hline FA-7-15 & 89 & 11,465 & 0.00108 & 0.282997 & 0.000009 & 7.96 & 0.282997 & 8.20 & 0.35 & 0.55 & 0.282765 & 0.283243 \\
\hline FA-7-17 & 122 & 15,691 & 0.00108 & 0.282985 & 0.000005 & 7.53 & 0.282985 & 7.78 & 0.37 & 0.58 & 0.282765 & 0.283243 \\
\hline FA-7-18 & 65 & 9,688 & 0.00093 & 0.282970 & 0.000006 & 7.00 & 0.282970 & 7.24 & 0.39 & 0.61 & 0.282765 & 0.283243 \\
\hline FA-7-19 & 89 & 11,565 & 0.00107 & 0.282995 & 0.000009 & 7.89 & 0.282995 & 8.15 & 0.35 & 0.56 & 0.282764 & 0.283242 \\
\hline FA-7-20 & 154 & 12,303 & 0.00175 & 0.283008 & 0.000008 & 8.35 & 0.283008 & 8.58 & 0.34 & 0.53 & 0.282765 & 0.283243 \\
\hline FA-7-21 & 52 & 11,214 & 0.00064 & 0.282999 & 0.000007 & 8.03 & 0.282999 & 8.28 & 0.34 & 0.55 & 0.282765 & 0.283242 \\
\hline FA-7-22 & 65 & 10,991 & 0.00083 & 0.283002 & 0.000008 & 8.13 & 0.283002 & 8.38 & 0.34 & 0.54 & 0.282765 & 0.283243 \\
\hline FA-8-01 & 64 & 11,094 & 0.00080 & 0.283006 & 0.000007 & 8.28 & 0.283006 & 8.60 & 0.34 & 0.53 & 0.282763 & 0.283240 \\
\hline FA-8-02 & 91 & 10,242 & 0.00124 & 0.283028 & 0.000007 & 9.05 & 0.283028 & 9.38 & 0.31 & 0.48 & 0.282762 & 0.283240 \\
\hline FA-8-03 & 63 & 11,047 & 0.00079 & 0.283003 & 0.000006 & 8.17 & 0.283003 & 8.50 & 0.34 & 0.54 & 0.282762 & 0.283240 \\
\hline FA-8-04 & 97 & 10,758 & 0.00126 & 0.283047 & 0.000006 & 9.73 & 0.283047 & 10.05 & 0.28 & 0.44 & 0.282762 & 0.283240 \\
\hline FA-8-05 & 40 & 12,824 & 0.00043 & 0.283014 & 0.000006 & 8.56 & 0.283014 & 8.88 & 0.32 & 0.51 & 0.282763 & 0.283240 \\
\hline FA-8-06 & 78 & 11,337 & 0.00096 & 0.283039 & 0.000007 & 9.44 & 0.283039 & 9.77 & 0.29 & 0.46 & 0.282763 & 0.283240 \\
\hline FA-8-07 & 76 & 13,716 & 0.00077 & 0.283031 & 0.000008 & 9.16 & 0.283031 & 9.49 & 0.30 & 0.48 & 0.282762 & 0.283240 \\
\hline FA-8-08 & 91 & 11,607 & 0.00109 & 0.283029 & 0.000008 & 9.09 & 0.283029 & 9.41 & 0.31 & 0.48 & 0.282763 & 0.283240 \\
\hline FA-8-09 & 71 & 11,497 & 0.00086 & 0.283020 & 0.000007 & 8.77 & 0.283020 & 9.10 & 0.32 & 0.50 & 0.282762 & 0.283240 \\
\hline FA- $8-10$ & 109 & 16,656 & 0.00091 & 0.283035 & 0.000006 & 9.30 & 0.283035 & 9.63 & 0.30 & 0.47 & 0.282762 & 0.283240 \\
\hline FA-8-11 & 144 & 10,580 & 0.00190 & 0.283113 & 0.000007 & 12.06 & 0.283112 & 12.38 & 0.19 & 0.30 & 0.282762 & 0.283240 \\
\hline FA-8-12 & 57 & 12,159 & 0.00066 & 0.283038 & 0.000007 & 9.41 & 0.283038 & 9.74 & 0.29 & 0.46 & 0.282763 & 0.283240 \\
\hline FA- $8-13$ & 79 & 11,079 & 0.00099 & 0.283045 & 0.000007 & 9.65 & 0.283045 & 9.98 & 0.28 & 0.45 & 0.282762 & 0.283240 \\
\hline FA-8-14 & 47 & 11,702 & 0.00056 & 0.283017 & 0.000007 & 8.66 & 0.283017 & 9.00 & 0.32 & 0.51 & 0.282762 & 0.283240 \\
\hline FA-8-15 & 55 & 13,185 & 0.00059 & 0.283039 & 0.000007 & 9.44 & 0.283039 & 9.77 & 0.29 & 0.46 & 0.282762 & 0.283240 \\
\hline FA-8-16 & 74 & 10,047 & 0.00102 & 0.283072 & 0.000008 & 10.61 & 0.283072 & 10.94 & 0.25 & 0.39 & 0.282762 & 0.283240 \\
\hline FA- $8-18$ & 58 & 10,492 & 0.00077 & 0.283045 & 0.000007 & 9.65 & 0.283045 & 9.98 & 0.28 & 0.45 & 0.282762 & 0.283240 \\
\hline FA-8-19 & 73 & 9,977 & 0.00102 & 0.283051 & 0.000006 & 9.87 & 0.283051 & 10.19 & 0.28 & 0.43 & 0.282763 & 0.283240 \\
\hline FA-8-20 & 45 & 10,904 & 0.00057 & 0.283038 & 0.000007 & 9.41 & 0.283038 & 9.75 & 0.29 & 0.46 & 0.282762 & 0.283240 \\
\hline FA-8-21 & 69 & 14,914 & 0.00064 & 0.283031 & 0.000008 & 9.16 & 0.283031 & 9.48 & 0.30 & 0.48 & 0.282763 & 0.283240 \\
\hline
\end{tabular}


TABLE 3d. Hf ISOTOPE COMPOSITIONS OF WHOLE ROCK AND ZIRCON MINERALS OF YERBA LOCA.

\begin{tabular}{|c|c|c|c|c|c|c|c|c|c|c|c|c|}
\hline & $\begin{array}{c}\text { Lu } \\
(\mathbf{p p m})\end{array}$ & $\begin{array}{c}\text { Hf } \\
(\mathbf{p p m})\end{array}$ & ${ }^{176} \mathbf{L u} /{ }^{177} \mathbf{H f}$ & $\left({ }^{176} \mathbf{H f} /{ }^{177} \mathbf{H f}\right) \mathbf{o}$ & $1 \sigma$ & $\varepsilon \mathbf{H f}(\mathbf{0})$ & $\left({ }^{176} \mathbf{H f} /{ }^{1{ }^{17}} \mathbf{H f}\right) \mathbf{i}$ & EHf(i) & $\begin{array}{l}\text { TDM } \\
(\mathbf{G a})\end{array}$ & $\begin{array}{c}\text { TDM } \\
\text { crustal } \\
(\text { Ga) }\end{array}$ & $\begin{array}{l}\text { Hf CHUR } \\
\text { (t) }\end{array}$ & Hf DM (t) \\
\hline \multicolumn{13}{|c|}{ WHOLE ROCK Yerba Loca (Central Andes, Chile) } \\
\hline FA-10 & 0.24 & 4.83 & 0.00706 & 0.282980 & 0.000002 & 7.36 & 0.282978 & 7.62 & 0.44 & 0.59 & 0.282762 & 0.283240 \\
\hline FA-11 & 0.23 & 3.76 & 0.00869 & 0.283020 & 0.000002 & 8.77 & 0.283018 & 9.02 & 0.40 & 0.51 & 0.282762 & 0.283240 \\
\hline \multicolumn{13}{|c|}{ ZIRCON Yerba Loca (Central Andes, Chile) } \\
\hline FA-9-01 & 84 & 10,332 & 0.00113 & 0.283037 & 0.000007 & 9.37 & 0.283037 & 9.70 & 0.30 & 0.46 & 0.282762 & 0.283240 \\
\hline FA-9-02 & 103 & 11,351 & 0.00126 & 0.283076 & 0.000006 & 10.75 & 0.283076 & 11.08 & 0.24 & 0.38 & 0.282762 & 0.283240 \\
\hline FA-9-03 & 155 & 13,219 & 0.00163 & 0.283106 & 0.000007 & 11.81 & 0.283106 & 12.13 & 0.20 & 0.31 & 0.282762 & 0.283240 \\
\hline FA-9-04 & 125 & 14,816 & 0.00117 & 0.283026 & 0.000006 & 8.98 & 0.283026 & 9.31 & 0.31 & 0.49 & 0.282762 & 0.283240 \\
\hline FA-9-05 & 124 & 11,274 & 0.00153 & 0.283104 & 0.000007 & 11.74 & 0.283104 & 12.07 & 0.20 & 0.32 & 0.282762 & 0.283240 \\
\hline FA-9-06 & 121 & 11,148 & 0.00150 & 0.283121 & 0.000010 & 12.34 & 0.283121 & 12.66 & 0.18 & 0.28 & 0.282763 & 0.283240 \\
\hline FA-9-07 & 101 & 14,543 & 0.00097 & 0.283064 & 0.000008 & 10.33 & 0.283064 & 10.65 & 0.26 & 0.40 & 0.282763 & 0.283240 \\
\hline FA-9-08 & 222 & 12,674 & 0.00244 & 0.283173 & 0.000009 & 14.18 & 0.283172 & 14.49 & 0.11 & 0.16 & 0.282763 & 0.283240 \\
\hline FA-9-09 & 94 & 13,820 & 0.00095 & 0.283052 & 0.000008 & 9.90 & 0.283052 & 10.23 & 0.27 & 0.43 & 0.282763 & 0.283240 \\
\hline FA-9-10 & 49 & 13,078 & 0.00052 & 0.283023 & 0.000008 & 8.88 & 0.283023 & 9.20 & 0.31 & 0.49 & 0.282763 & 0.283240 \\
\hline FA-11-01 & 230 & 14,012 & 0.00229 & 0.283114 & 0.000008 & 12.09 & 0.283113 & 12.41 & 0.19 & 0.29 & 0.282762 & 0.283240 \\
\hline FA-11-02 & 190 & 10,987 & 0.00240 & 0.283197 & 0.000011 & 15.03 & 0.283196 & 15.34 & 0.08 & 0.11 & 0.282762 & 0.283240 \\
\hline FA-11-03 & 277 & 11,473 & 0.00337 & 0.283189 & 0.000011 & 14.75 & 0.283188 & 15.06 & 0.09 & 0.13 & 0.282762 & 0.283240 \\
\hline FA-11-05 & 134 & 10,935 & 0.00170 & 0.283085 & 0.000007 & 11.07 & 0.283085 & 11.39 & 0.23 & 0.36 & 0.282762 & 0.283240 \\
\hline FA-11-06 & 235 & 11,360 & 0.00288 & 0.283190 & 0.000009 & 14.78 & 0.283189 & 15.08 & 0.09 & 0.13 & 0.282763 & 0.283240 \\
\hline FA-11-08 & 261 & 12,223 & 0.00297 & 0.283176 & 0.000008 & 14.29 & 0.283175 & 14.60 & 0.11 & 0.16 & 0.282762 & 0.283240 \\
\hline FA-11-09 & 259 & 11,222 & 0.00321 & 0.283190 & 0.000012 & 14.78 & 0.283189 & 15.09 & 0.09 & 0.13 & 0.282763 & 0.283240 \\
\hline FA-11-11 & 225 & 11,122 & 0.00281 & 0.283149 & 0.000010 & 13.33 & 0.283148 & 13.65 & 0.15 & 0.22 & 0.282762 & 0.283240 \\
\hline FA-11-13 & 130 & 9,682 & 0.00187 & 0.283122 & 0.000009 & 12.38 & 0.283121 & 12.69 & 0.18 & 0.28 & 0.282763 & 0.283240 \\
\hline FA-11-14 & 214 & 11,040 & 0.00270 & 0.283138 & 0.000009 & 12.94 & 0.283137 & 13.25 & 0.16 & 0.24 & 0.282762 & 0.283240 \\
\hline
\end{tabular}

Initial and epsilon values calculated using parameters after Blichert-Toft et al. (1997).

Since there is no significant variation in the ${ }^{176} \mathrm{Hf} /{ }^{177} \mathrm{Hf}$ ratio data set for the samples from Cerro Mesón Alto, La Gloria pluton and the youngest San Francisco Batholith, magma mixing or inheritance do not seem to have been important. The older samples (FA-8, -9, -11) however, do show a slightly greater isotopic variation, but no analyzed zircon grain shows evidence of older nuclei (Figs. 2-3) or reveals inherited U-Pb ages (Tables 2a-d).

\section{Whole rock geochemical characteristics and Sr-Nd-Hf systematics}

\subsection{Major and trace element characteristics}

Major and trace elements are presented in table 4 and figures 4-9. The Cerro Mesón Alto massif
(66-67 wt $\% \mathrm{SiO}_{2}$ ), La Gloria pluton (61.6-67 wt $\%$ $\left.\mathrm{SiO}_{2}\right)$. Yerba Loca stock (54-57.5 wt $\% \mathrm{SiO}_{2}$ ) and San Fancisco Batholith (56.3-62.4 wt $\% \mathrm{SiO}_{2}$ ) late Miocene plutonic rocks show a calc-alkaline to high-K calc-alkaline and metaluminous character. All analyzed rocks fall in the quartz monzodiorite and quartz monzogabbro fields after Streckeisen (1976).

Low $\mathrm{MgO}(<2.5 \mathrm{wt} \%)$, $\mathrm{Ni}$ and $\mathrm{Cr}$ concentrations from the Cerro Mesón Alto massif and La Gloria pluton are indicative of their fractionated character. The San Francisco Batholith and Yerba Loca igneous rocks show higher $\mathrm{MgO}(>2.5 \mathrm{wt} \%), \mathrm{Ni}$ and $\mathrm{Cr}$ contents compared to the others. There is an overall increase in transition metal element $(\mathrm{Cu}$, $\mathrm{Cr}, \mathrm{Co}, \mathrm{Ni}, \mathrm{V}, \mathrm{Zn}, \mathrm{Sc}$ ) concentrations in the more mafic lithologies and at decreasing distance to the mineral district Río Blanco-Los Bronces. FA-8 from 
TABLE 4. MAJOR AND TRACE ELEMENT COMPOSITIONS AND AGES OF CMA, LGP, SFB AND YL BARREN INTRUSIVES.

\begin{tabular}{|c|c|c|c|c|c|c|c|c|c|c|c|}
\hline \multicolumn{4}{|c|}{ Cerro Mesón Alto (CMA) } & \multicolumn{3}{|c|}{ La Gloria pluton (LGP) } & \multicolumn{3}{|c|}{ San Francisco Batholith (SFB) } & \multicolumn{2}{|c|}{ Yerba Loca (YL) } \\
\hline Sample & FA-1 & FA-3 & FA-3a & FA-4 & FA-5 & FA-6 & FA-7 & FA-7a & FA-8 & FA-10 & FA-11 \\
\hline $\mathrm{SiO}_{2}$ & 66.57 & 66.83 & - & 61.61 & 66.84 & 63.02 & 62.17 & 62.41 & 56.29 & 57.51 & 54.01 \\
\hline $\mathrm{TiO}_{2}$ & 0.43 & 0.43 & - & 0.62 & 0.45 & 0.62 & 0.77 & 0.78 & 1.14 & 0.98 & 0.95 \\
\hline $\mathrm{Al}_{2} \mathrm{O}_{3}$ & 15.99 & 15.60 & - & 16.84 & 15.66 & 16.19 & 16.07 & 16.05 & 16.45 & 17.14 & 17.49 \\
\hline $\mathrm{Fe}_{2} \mathrm{O}_{3}$ & 3.31 & 3.71 & - & 5.31 & 3.56 & 4.83 & 5.15 & 5.13 & 8.02 & 6.88 & 7.80 \\
\hline $\mathrm{MnO}$ & 0.03 & 0.03 & - & 0.09 & 0.04 & 0.06 & 0.05 & 0.05 & 0.13 & 0.12 & 0.11 \\
\hline $\mathrm{MgO}$ & 1.24 & 1.28 & - & 2.39 & 1.53 & 2.35 & 2.68 & 2.69 & 3.81 & 3.16 & 4.35 \\
\hline $\mathrm{CaO}$ & 2.99 & 3.03 & - & 5.11 & 3.17 & 4.67 & 4.41 & 4.39 & 5.88 & 6.10 & 7.06 \\
\hline $\mathrm{Na}_{2} \mathrm{O}$ & 5.28 & 4.80 & - & 4.70 & 4.69 & 4.61 & 4.62 & 4.62 & 4.21 & 4.46 & 4.27 \\
\hline $\mathrm{K}_{2} \mathrm{O}$ & 2.92 & 3.19 & - & 2.68 & 3.42 & 2.91 & 2.91 & 2.94 & 2.59 & 2.44 & 2.04 \\
\hline $\mathrm{P}_{2} \mathrm{O}_{5}$ & 0.12 & 0.12 & - & 0.19 & 0.11 & 0.15 & 0.17 & 0.18 & 0.30 & 0.25 & 0.24 \\
\hline LOI & 1.07 & 0.88 & - & 0.34 & 0.44 & 0.48 & 0.66 & 0.66 & 1.03 & 0.82 & 1.56 \\
\hline TOTAL & 99.94 & 99.90 & - & 99.87 & 99.91 & 99.88 & 99.66 & 99.90 & 99.84 & 99.86 & 99.87 \\
\hline $\mathrm{Sc}$ & 4.7 & 5.5 & 5.6 & 10.3 & 6.2 & 9.8 & 12.1 & - & 18.6 & 15.7 & 17.8 \\
\hline V & 54 & 54 & - & 124 & 68 & 119 & 117 & - & 180 & 180 & 191 \\
\hline $\mathrm{Cr}$ & 2 & 4 & - & 24 & 21 & 29 & 55 & - & 58 & 17 & 51 \\
\hline $\mathrm{Co}$ & 6 & 6 & - & 12 & 8 & 10 & 15 & - & 20 & 13 & 22 \\
\hline $\mathrm{Ni}$ & 3 & 4 & - & 11 & 9 & 16 & 26 & - & 27 & 21 & 35 \\
\hline $\mathrm{Cu}$ & 6 & 6 & - & 34 & 5 & 10 & 13 & - & 204 & 89 & 88 \\
\hline $\mathrm{Zn}$ & 14 & 15 & 15 & 47 & 21 & 31 & 47 & - & 90 & 89 & 71 \\
\hline $\mathrm{Ga}$ & 15.5 & 16.4 & 16.3 & 16.7 & 16.9 & 16.8 & 21.6 & - & 21.0 & 23.5 & 21.5 \\
\hline $\mathrm{Ge}$ & 1.5 & 1.6 & - & 1.2 & 1.5 & 1.1 & 1.3 & - & 1.4 & 1.2 & 1.2 \\
\hline $\mathrm{Rb}$ & 51 & 60 & 61 & 65 & 99 & 70 & 96 & - & 109 & 113 & 91 \\
\hline $\mathrm{Sr}$ & 337 & 338 & 338 & 569 & 375 & 504 & 504 & - & 489 & 528 & 599 \\
\hline $\mathrm{Y}$ & 10.6 & 11.4 & 11.6 & 13.6 & 10.3 & 14.1 & 14.9 & - & 24.4 & 18.9 & 17.0 \\
\hline $\mathrm{Zr}$ & 5.9 & 6.3 & 5.7 & 8.5 & 6.4 & 13.7 & 220 & - & 218 & 201 & 143 \\
\hline $\mathrm{Nb}$ & 5.0 & 5.3 & 5.4 & 4.5 & 5.2 & 5.7 & 6.4 & - & 8.5 & 7.5 & 5.4 \\
\hline Mo & 0.1 & 0.1 & 0.1 & 0.2 & 0.2 & 0.3 & 3.8 & - & 4.8 & 1.5 & 0.8 \\
\hline Sn & 0.8 & 0.9 & 0.9 & 1.4 & 1.3 & 1.7 & 1.8 & - & 2.5 & 2.1 & 1.6 \\
\hline $\mathrm{Sb}$ & 0.4 & 0.4 & 0.4 & 0.4 & 0.3 & 0.4 & - & - & 1.1 & - & 2.9 \\
\hline Cs & 0.4 & 0.6 & 0.6 & 2.6 & 2.4 & 1.3 & 1.4 & - & 2.5 & 5.0 & 5.8 \\
\hline $\mathrm{Ba}$ & 497 & 541 & 531 & 501 & 558 & 513 & 489 & - & 423 & 411 & 371 \\
\hline $\mathrm{La}$ & 18.9 & 26.4 & 27.1 & 17.0 & 13.2 & 18.7 & 18.4 & - & 23.0 & 20.0 & 15.8 \\
\hline $\mathrm{Ce}$ & 36.2 & 48.8 & 50.4 & 37.6 & 29.2 & 42.0 & 44.4 & - & 52.2 & 46.9 & 37.0 \\
\hline $\operatorname{Pr}$ & 4.30 & 5.44 & 5.58 & 4.87 & 3.77 & 5.54 & 5.42 & - & 6.76 & 5.83 & 4.67 \\
\hline $\mathrm{Nd}$ & 16.0 & 18.4 & 19.0 & 19.3 & 14.4 & 21.1 & 21.1 & - & 27.8 & 22.2 & 18.6 \\
\hline $\mathrm{Sm}$ & 2.99 & 3.12 & 3.15 & 3.82 & 2.79 & 4.07 & 3.59 & - & 5.51 & 4.36 & 3.68 \\
\hline $\mathrm{Eu}$ & 0.87 & 0.90 & 0.90 & 1.07 & 0.83 & 1.08 & 0.91 & - & 1.25 & 1.09 & 1.05 \\
\hline Gd & 2.77 & 3.11 & 3.15 & 3.45 & 2.55 & 3.66 & 3.38 & - & 4.85 & 3.78 & 3.62 \\
\hline Dy & 1.91 & 2.01 & 2.02 & 2.40 & 1.80 & 2.54 & 2.69 & - & 4.27 & 3.41 & 2.76 \\
\hline Но & 0.39 & 0.41 & 0.41 & 0.49 & 0.37 & 0.52 & 0.50 & - & 0.82 & 0.65 & 0.61 \\
\hline $\mathrm{Er}$ & 1.11 & 1.17 & 1.18 & 1.37 & 1.04 & 1.44 & 1.47 & - & 2.39 & 2.08 & 1.93 \\
\hline $\mathrm{Yb}$ & 1.07 & 1.16 & 1.16 & 1.28 & 0.98 & 1.36 & 1.36 & - & 1.92 & 1.75 & 1.58 \\
\hline $\mathrm{Lu}$ & 0.15 & 0.17 & 0.17 & 0.19 & 0.14 & 0.20 & 0.19 & - & 0.29 & 0.24 & 0.23 \\
\hline Hf & 0.31 & 0.31 & 0.28 & 0.59 & 0.34 & 0.72 & 5.39 & - & 5.33 & 4.83 & 3.76 \\
\hline $\mathrm{Ta}$ & 0.44 & 0.48 & 0.52 & 0.31 & 0.42 & 0.41 & 0.59 & - & 0.67 & 0.61 & 0.42 \\
\hline $\mathrm{Pb}$ & 3.75 & 4.26 & 4.25 & 8.31 & 6.96 & 7.28 & 15.5 & - & 18.5 & 19.8 & 30.4 \\
\hline $\mathrm{Th}$ & 14.2 & 14.6 & 14.2 & 10.3 & 12.8 & 10.0 & 8.9 & - & 9.0 & 9.0 & 7.2 \\
\hline $\mathrm{U}$ & 1.31 & 1.41 & 1.36 & 2.67 & 1.43 & 3.04 & 11.3 & - & 5.7 & 3.5 & 2.3 \\
\hline $\mathrm{Sr} / \mathrm{P}(\mathrm{n})$ & 3.67 & 3.63 & - & 3.96 & 4.44 & 4.46 & 3.83 & - & 2.11 & 2.76 & 3.22 \\
\hline $\mathrm{La} / \mathrm{Ta}$ & 43.5 & 54.5 & 52.1 & 31.2 & 54.5 & 45.3 & 31.2 & - & 34.3 & 32.8 & 37.6 \\
\hline $\mathrm{Ba} / \mathrm{La}$ & 26.2 & 20.5 & 19.5 & 29.4 & 42.3 & 27.4 & 26.6 & - & 18.4 & 20.6 & 23.5 \\
\hline $\mathrm{La} / \mathrm{Yb}$ & 17.7 & 22.8 & 23.4 & 13.4 & 13.3 & 13.8 & 13.5 & - & 12.0 & 11.4 & 10.0 \\
\hline $\mathrm{Th} / \mathrm{U}$ & 10.82 & 10.35 & 10.46 & 3.87 & 8.96 & 3.29 & 0.79 & - & 1.58 & 2.57 & 3.13 \\
\hline Age (Ma) & & \pm 0.10 & & 10. & 0.15 & & $11.16 \pm 0.07$ & & $14.74 \pm 0.13$ & & $14.94 \pm 0.14$ \\
\hline UTM & $\begin{array}{l}\text { N6.276.2 } \\
\text { E401.432 }\end{array}$ & & & $\begin{array}{l}\text { N6.293.008 } \\
\text { E392.427 }\end{array}$ & & $\begin{array}{l}\text { N6.293.338- } \\
\text { E391.979 }\end{array}$ & $\begin{array}{l}\text { N6.330.797- } \\
\text { E376.951 }\end{array}$ & & $\begin{array}{l}\text { N6.322.915- } \\
\text { E375.119 }\end{array}$ & $\begin{array}{l}\text { N6.315.231- } \\
\text { E377.608 }\end{array}$ & $\begin{array}{l}\text { N6.315.160- } \\
\text { E377.445 }\end{array}$ \\
\hline
\end{tabular}



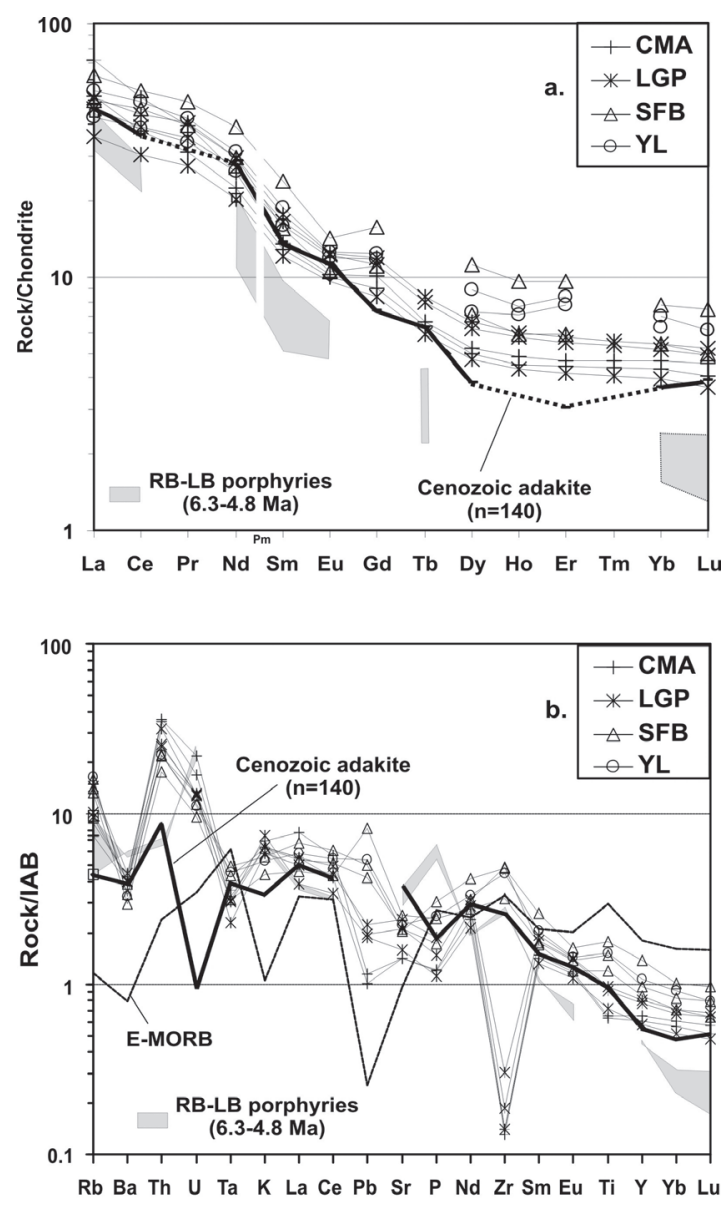

FIG. 4. a. REE plot obtained for new rock data normalized to chondrite (Sun and McDonough, 1989) and, as reference, Río Blanco-Los Bronces porphyries (6.3-4.8 Ma) and average Cenozoic adakite ( $\mathrm{n}=140)$ (Drummond $e t$ al.,1996); b. trace multi-element diagram normalized to Island Arc Basalts (IAB; Ewart et al., 1998) with E-MORB average values (Niu and O'Hara, 2003), Río Blanco-Los Bronces porphyries (6.3-4.8 Ma) and average Cenozoic adakite $(\mathrm{n}=140)$ (Drummond et al., 1996) as reference.

the San Francisco Batholith has the highest $\mathrm{Cu}, \mathrm{Mo}$, $\mathrm{Sc}$ and $\mathrm{Cr}$ content.

A slightly negative-to-no-Eu anomaly (Fig. 4) and $\mathrm{CaO} / \mathrm{Al}_{2} \mathrm{O}_{3}$ ratios correlated with Sc, suggest clinopyroxene fractionation in all samples. Rare earth element (REE) plots show a 60- to 100 -fold enrichment in light REE, around 20-fold enrichment for middle REE and a 5- to 7-fold enrichment for heavy REE relative to chondritic values (Fig. 4a). The slight enrichment of the heavy REE relative to chondrite suggests that amphibole and possibly garnet might be minor residual phases in the magma source. $(\mathrm{La} / \mathrm{Yb})_{\mathrm{n}}$ ratios range from 11.95 to 15.34 in the Cerro Mesón Alto massif, 8.96 to 9.33 in the La Gloria pluton, 6.73 to 7.76 in the Yerba Loca stock, and 8.07 to 9.16 in the San Francisco Batholith. Cerro Meson Alto and La Gloria have a higher potassic and $\mathrm{SiO}_{2}$ content indicating a higher degree of fractionation. Similar major and rare earth element observations have been outlined in Kay et al. (2005) on whole rock geochemical analyses of contemporaneous rock units.

Trace element patterns are typical of continental margin subduction-related arc magmas. The samples are enriched (2-38 times) in large ion lithophile elements (LILE; e.g., Rb, Ba, Th, U, Ta, K, La, Ce) relative to island arc basalts (IAB; Ewart et al., 1998; Fig. 4b). High field strength elements (HFSE), Ti, $\mathrm{Sr}$, and the heavy REEs show similar and/or slight enrichment or depletion relative to IAB. The strong negative anomaly for $\mathrm{Zr}$ and likewise for $\mathrm{Hf}$ (not shown) and $\mathrm{Pb}$ (Cerro Mesón Alto massif, La Gloria pluton) may be due to incomplete analytical acid digestion of zircon minerals. Differences between $\mathrm{IAB}$ and the active continental margin basalts are seen in element concentrations such as $\mathrm{K}, \mathrm{Sr}, \mathrm{Rb}, \mathrm{Ba}$, $\mathrm{Zr}$, Th and $\mathrm{U}$, which are more enriched in the latter.

The same rock concentrations normalized to average N-MORB show an even stronger enrichment in more or less the same elements $(\mathrm{Rb}, \mathrm{Ba}$, $\mathrm{Sr}, \mathrm{Th}, \mathrm{U}, \mathrm{K}, \mathrm{Nd}, \mathrm{La}, \mathrm{Ce}$ and $\mathrm{Pb}$ ) compared to the IAB normalization and confirm a LILE transfer to the magma source area. Enrichment of these elements is usually explained through partial melts in the mantle wedge or fluids liberated from the subducted slab.

In order to compare and enlarge the major and trace element data set for the Middle-to-Late Miocene granitoids of the Central Andes, published information was included. Geochemical data of four Coya Machalí (CM) and three Farellones Formation (FF) volcanic rocks from Kay et al. (2005) were included since it has been suggested that coeval igneous rocks originated from the same magma source (e.g., López-Escobar et al., 1979; Nyström et al., 2003). A sample from the La Obra Plutonic Complex (LO; ca. 20 Ma), three (Lago Yeso, Melosas, San Gabriel) from the older Teniente Plutonic Complex (12-8 Ma; TPC-1; Kay et al., 2005) and three (Cerro Cortaderal, Carlota, Extravío) from the younger Teniente Plutonic Complex (7-3 Ma; TPC-2; Kay et al., 2005) along with five samples 
from mineralized porphyritic intrusive units at Río Blanco-Los Bronces Cu(-Mo) deposit (RB-LB; Stern and Skewes, 1995); age range of mineralized porphyries (6.3-4.8 Ma; Warnaars et al., 1985; Deckart et al., 2005) were also included.

Harker diagrams of oxides versus $\mathrm{SiO}_{2}$ content (Fig. 5a-h) show a negative correlation of $\mathrm{Al}_{2} \mathrm{O}_{3}$, $\mathrm{MgO}, \mathrm{CaO}, \mathrm{FeO}^{*}, \mathrm{TiO}_{2}$ and $\mathrm{P}_{2} \mathrm{O}_{5}$ with increasing $\mathrm{SiO}_{2}$ concentrations and an overall positive correlation of $\mathrm{Na}_{2} \mathrm{O}$ and $\mathrm{K}_{2} \mathrm{O}$ versus $\mathrm{SiO}_{2}$ for the barren magmatism. Major element patterns are similar for the fertile rock sample group from the Río Blanco-
Los Bronces deposit except for $\mathrm{Na}_{2} \mathrm{O}$ indicating a negative correlation with an increasing $\mathrm{SiO}_{2}$ content. Barren andesites from the Coya Machalí and Farellones formations are characterized by the lowest $\mathrm{SiO}_{2}$ content (50-61 wt\%), followed by the barren igneous groups (58-71 wt\%) without any tendency within the rock groups. The Río BlancoLos Bronces porphyric rock group shows the highest $\mathrm{SiO}_{2}$ content ranging from 66 to $71 \mathrm{wt} \%$.

Trace elements $(\mathrm{Ni}, \mathrm{Sc}, \mathrm{Cr})$ versus $\mathrm{SiO}_{2}$ content (Fig. 6a-e) show a negative correlation, whereas $\mathrm{Ba}$ indicates a positive pattern and $\mathrm{Sr}$ yields little

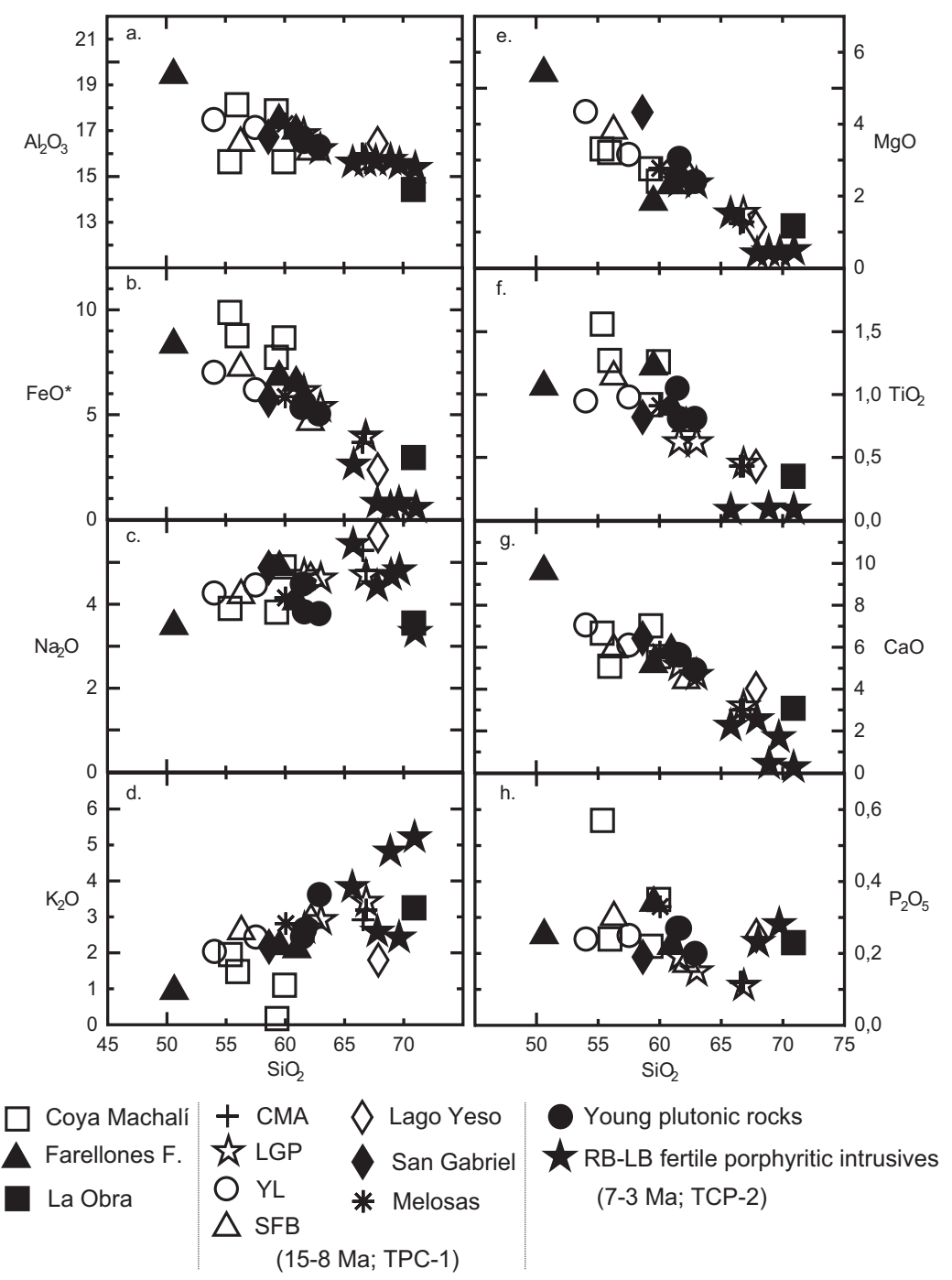

FIG. 5. Major element (wt\%) Harker diagrams with new obtained data and selected data from Kay et al. (2005) and Stern and Skewes (1995). a. $\mathrm{Al}_{2} \mathrm{O}_{3}$; b. $\mathrm{FeO}^{*}$; c. $\mathrm{Na}_{2} \mathrm{O}$; d. $\mathrm{K}_{2} \mathrm{O}$; e. $\mathrm{MgO}$; f. $\mathrm{TiO}_{2}$; g. $\mathrm{CaO}$ and, h. $\mathrm{P}_{2} \mathrm{O}_{5}$ versus $\mathrm{SiO}_{2}$ diagrams. TPC-1 and TPC-2 plutonic groups are adapted to the herein used age data set. 
correlation for the andesitic and barren plutonic rock groups. Porphyric Río Blanco-Los Bronces rocks (LOI: $0.85-2.0 \mathrm{wt} \%$ ) show a positive correlation for $\mathrm{Ba}$, a negative for $\mathrm{Sr}$ and rather no correlation for $\mathrm{Sc}$ with increasing $\mathrm{SiO}_{2}$ content. $(\mathrm{La} / \mathrm{Eu})_{\mathrm{n}}$ ratios versus $\mathrm{La}_{\mathrm{n}}$ concentrations (normalization after Sun and McDonough, 1989) rules out a break between volcanic and plutonic rocks, with ratios under 3 for the former and greater than 3 for the latter at a similar $\mathrm{La}_{\mathrm{n}}$ concentration (Fig. 7a). Río Blanco-Los Bronces porphyric rocks show the highest $(\mathrm{La} / \mathrm{Yb})_{\mathrm{n}}$ ratios (14-28), implying either a strongly fractionated character or possible residual garnet in the source of this group (Fig. 4). These major and trace elements show that the volcanics are less differentiated than the coeval to younger barren and fertile porphyric rocks, which requires differences in magma source regions and/or differences in the entire tectonomagmatic framework at the time of their magma formation.

The trace elements ratios $\mathrm{Ba} / \mathrm{Th}$ versus $\mathrm{Ta} / \mathrm{Yb}$ (Pearce et al., 2005; Fig. 8a) clearly separate the herein selected volcanic from the plutonic barren groups. The higher $\mathrm{Ba} / \mathrm{Th}$ ratio of the volcanic group (low $\mathrm{Ta} / \mathrm{Yb}$ ) may be linked to a low-temperature fluid component, whereas all barren plutonic groups have a higher $\mathrm{Ta} / \mathrm{Yb}$ with lower $\mathrm{Ba} / \mathrm{Th}$ ratios indicating an enriched mantle influence (N-MORB: $\mathrm{Ta} / \mathrm{Yb}=0.04 ; \mathrm{Ba} / \mathrm{Th}=52.5$; Sun and McDonough, 1989) and/or recycled terrigeneous sediments rather than a shallow subduction (low-T, Ba-bearing, no significant Th addition) component (Pearce et al., 2005). In contrast, Kay et al. (2005) demonstrated that the Teniente Volcanic Complex group has similar characteristics with plutons of the immediate vicinity of El Teniente area. In the trace element ratio diagram $(\mathrm{La} / \mathrm{Yb}$ versus $\mathrm{Ba} / \mathrm{La}$; Fig. $8 \mathrm{~b})$ the high $\mathrm{Ba} / \mathrm{La}$ and low $\mathrm{La} / \mathrm{Yb}$ ratios suggest rather dominating subduction fluids (i.e., La Obra and $\mathrm{La}$ Gloria plutons) during genesis (e.g., Tollstrup and Gill, 2005; Elliott, 2003), whereas a low Ba/La with increasing $\mathrm{La} / \mathrm{Yb}$ ratios (Cerro Mesón Alto massif, San Francisco Batholith, and Yerba Loca stock, and 12-8 Ma Teniente Plutonic Complex [TPC-1]) might indicate a slightly increasing terrigenous sediment involvement during genesis. Río Blanco-Los Bronces porphyric intrusives show a tendency to both, slightly elevated $\mathrm{La} / \mathrm{Yb}$ and $\mathrm{Ba} / \mathrm{La}$ ratios pointing to a more complex magma genesis.

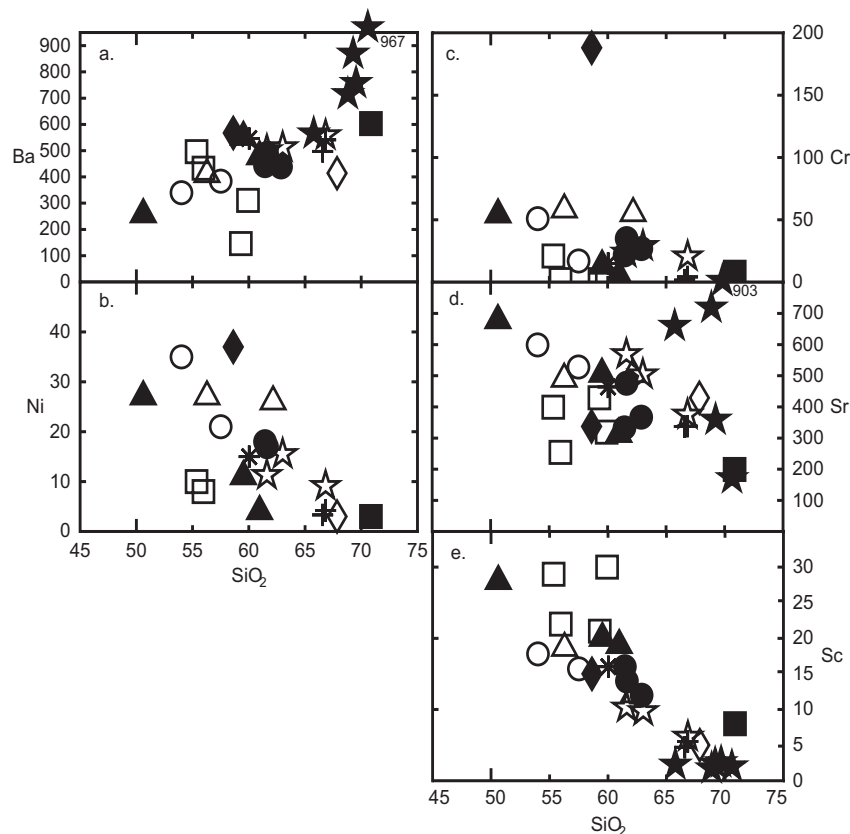

$$
\begin{aligned}
& \text { Coya Machalí + CMA } \diamond \text { Lago Yeso } \\
& \text { Farellones F. 疋 LGP } \\
& \text { La Obra } \\
& \bigcirc \mathrm{YL} \\
& \triangle \text { SFB } \\
& \text { San Gabriel } \\
& \text { * Melosas } \\
& \star x
\end{aligned}
$$

FIG. 6. Trace element (ppm) Harker diagrams with new obtained data and selected data from Kay et al. (2005) and Stern and Skewes (1995). a. Ba; b. Ni; c. Cr; d. $\mathrm{Sr}$ and e. Sc versus $\mathrm{SiO}_{2}(\mathrm{wt} \%)$ diagrams. 


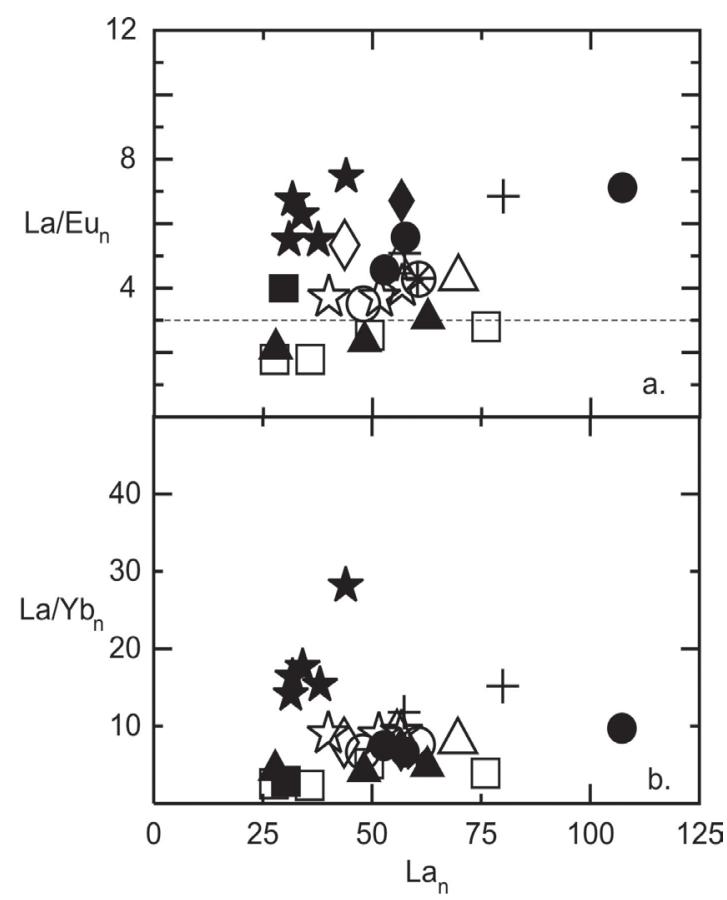

FIG. 7. a. $\mathrm{La}_{\mathrm{n}}$ versus $\mathrm{La} / \mathrm{Eu}_{\mathrm{n}} ; \mathbf{b}$. $\mathrm{La}_{\mathrm{n}}$ versus $\mathrm{La} / \mathrm{Yb}_{\mathrm{n}}$. Normalization after Sun and McDonough (1989). Symbols as in figure 6.

$(\mathrm{Sr} / \mathrm{P})_{\mathrm{n}}$ ratios ranging from 3.63 to 4.46 for the Cerro Mesón Alto massif and La Gloria pluton and from 2.11 to 3.83 for the San Francisco Batholith and Yerba Loca stock, suggest enrichment through a fluid component rather than sediment influence (Borg et al., 1997). Pelagic and ODP Leg 141 sediments (Kilian and Behrmann, 2003) yield ( $\mathrm{Sr} / \mathrm{P})_{\mathrm{n}}$ ratios of 1.65 and 1.43 , respectively. On the other hand, the higher $\mathrm{Pb} / \mathrm{La}$ ratio (about 1-2) in the Yerba Loca stock could suggest a possible sediment influence during genesis or slight crustal contamination by an elevated $\mathrm{Pb}$ signature in the older magmatic group. Trace element ratios of $\mathrm{La} / \mathrm{Ta}>25$ (Hickey et al., 1986) indicate a typical arc-like signature for all available data from the here presented rock units. Furthermore, ratios such as $\mathrm{Th} / \mathrm{Yb}$ and $\mathrm{Ta} /$ $\mathrm{Yb}$ usually indicate the involvement of an enriched mantle source with a slight subduction component and/or slight crustal contamination during the genesis of these magmas, but do not discriminate unambiguously between them (Pearce, 1983).

A slightly distinct evolution regarding the younger and slightly older plutonic rock groups may however be suggested considering the trace element ratios reported in this paper. Fluid dominance related to subduction processes are recognized in Cerro Mesón
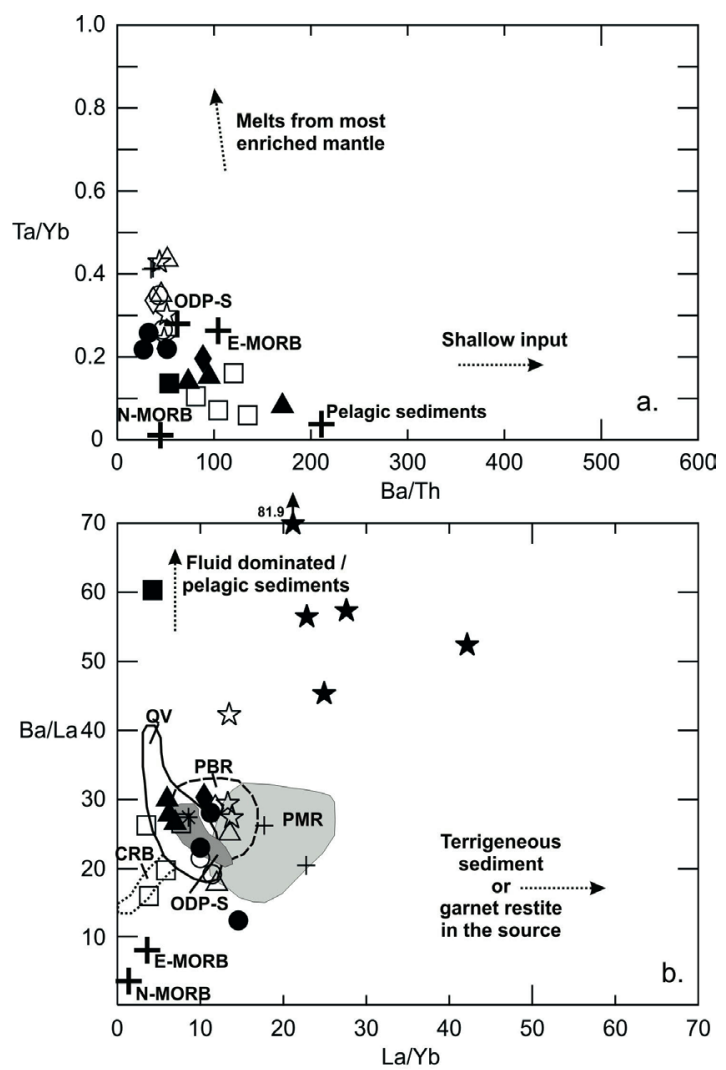

FIG. 8. Trace element ratio plots showing tendencies of involved material during magma genesis, a. $\mathrm{Ba} / \mathrm{Th}$ versus $\mathrm{Ta} / \mathrm{Yb}$ and, b. $\mathrm{La} / \mathrm{Yb}$ versus $\mathrm{Ba} / \mathrm{La}$ with compositional fields after Kilian and Behrmann (2003) (QV: Quaternary Volcanics; ODP-S: ODP-Leg 141 sediments; CRB: Chilean Rise basalts; PBR: Patagonian Batholith rocks; PMR: Paleozoic metasedimentary rocks). Presented are new obtained data and selected data from Kay et al. (2005) and Río Blanco-Los Bronces rocks (Stern and Skewes, 1995), average sediment compositions from Kilian and Behrmann (2003), N-MORB and E-MORB (Niu and O'Hara, 2003). Symbols as in figure 6.

Alto and La Gloria pluton whereas sediment influence is manifested in magma evolution in the Yerba Loca stock and possibly the San Francisco Batholith.

\subsection{Isotopic characteristics}

Whole rock $\mathrm{Sr}, \mathrm{Nd}$ and $\mathrm{Hf}$ isotope ratios were measured in two samples from the Cerro Mesón Alto massif, three from La Gloria pluton, two from the Yerba Loca stock and two from the San Francisco Batholith (Tables 3-5). Initial ratios were calculated back to the individual intrusive ages ranging between 15-10 Ma. Since the number of samples involved is 
rather small, isotope modelling was not attempted. Instead, isotopic ratios are plotted in conventional diagrams or combined with trace element ratios (Figs. 9-11).

Initial Sr- and Nd-isotope ratios $(0.703761$ 0.704118 and $0.512758-0.512882$ ) show a restricted range for all four intrusions of the study area. The similarity in Hf-isotope ratios for whole rock and zircons, probably especially in the case of the younger intrusive rocks, indicates that zircon is most possibly controlling the Hf-isotope values.

Isotope diagrams show that initial Nd-Sr isotopic ratios of the new data plot in the mantle array between Depleted Mantle (DM) and Bulk Silicate Earth (BSE) (Fig. 9). The southern intrusives (Cerro Mesón Alto and La Gloria pluton) are slightly more radiogenic in their ${ }^{87} \mathrm{Sr} /{ }^{86} \mathrm{Sr}_{i}$ ratio than the northern group (San Francisco Batholith and Yerba Loca stock) at equivalent ${ }^{143} \mathrm{Nd} /{ }^{144} \mathrm{Nd}_{\mathrm{i}}$ values. As a result of this characteristic the southern group plots inside many fields: the Chilean Coastal Batholith of central Chile $\left(31^{\circ}-34^{\circ} \mathrm{S}\right)$ (Parada et al., 1999), where its depleted portion overlaps with the SSVZ volcanic field (Kilian and Behrmann, 2003), Teniente Volcanic Complex (Kay et al., 2005) and in parts with the Farellones and Abanico formations (Nyström et al., 2003). Río Blanco-Los Bronces porphyry (Stern and Skewes, $1995){ }^{87} \mathrm{Sr}^{86} \mathrm{Sr}_{i}$ ratios are similar with the SSVZ volcanic field but ${ }^{143} \mathrm{Nd} /{ }^{144} \mathrm{Nd}_{\mathrm{i}}$ values are less depleted compared to the rest of the rock groups of the depleted quadrant.

$\mathrm{Ba} / \mathrm{Nb}$ versus $\mathrm{Sr}$ and $\mathrm{Nd}$ initial isotopic compositions confirm the relative stability of the Sm$\left.\mathrm{Nd}\left({ }^{143} \mathrm{Nd} /{ }^{144} \mathrm{Nd}\right)_{\mathrm{i}}: 0.512758-0.512882\right)$ system in a subduction environment and suggests a fluid dominated process (increased $\mathrm{Ba} / \mathrm{Nb}$ ) for Cerro Mesón Alto, La Gloria pluton and Río Blanco-Los Bronces porphyric intrusives compared to the San Francisco Batholith and Yerba Loca stock (Fig. 10a-b).

The slightly wider range of the $\mathrm{Sr}$ isotope ratios compared to $\mathrm{Nd}$ might be related to higher $\mathrm{Rb}$ mobility during subduction processes and the robustness of the Sm-Nd based isotopic system. In the initial ${ }^{143} \mathrm{Nd} /{ }^{144} \mathrm{Nd}$ versus $\mathrm{Th} /$ La diagram, the data (Fig. 10c) plot mainly at an average SSVZ volcanics isotopic ratio but at higher $\mathrm{Th} / \mathrm{La}$ values. Exeptions are two samples, Cerro Mesón Alto and La Gloria pluton, which are characterized by

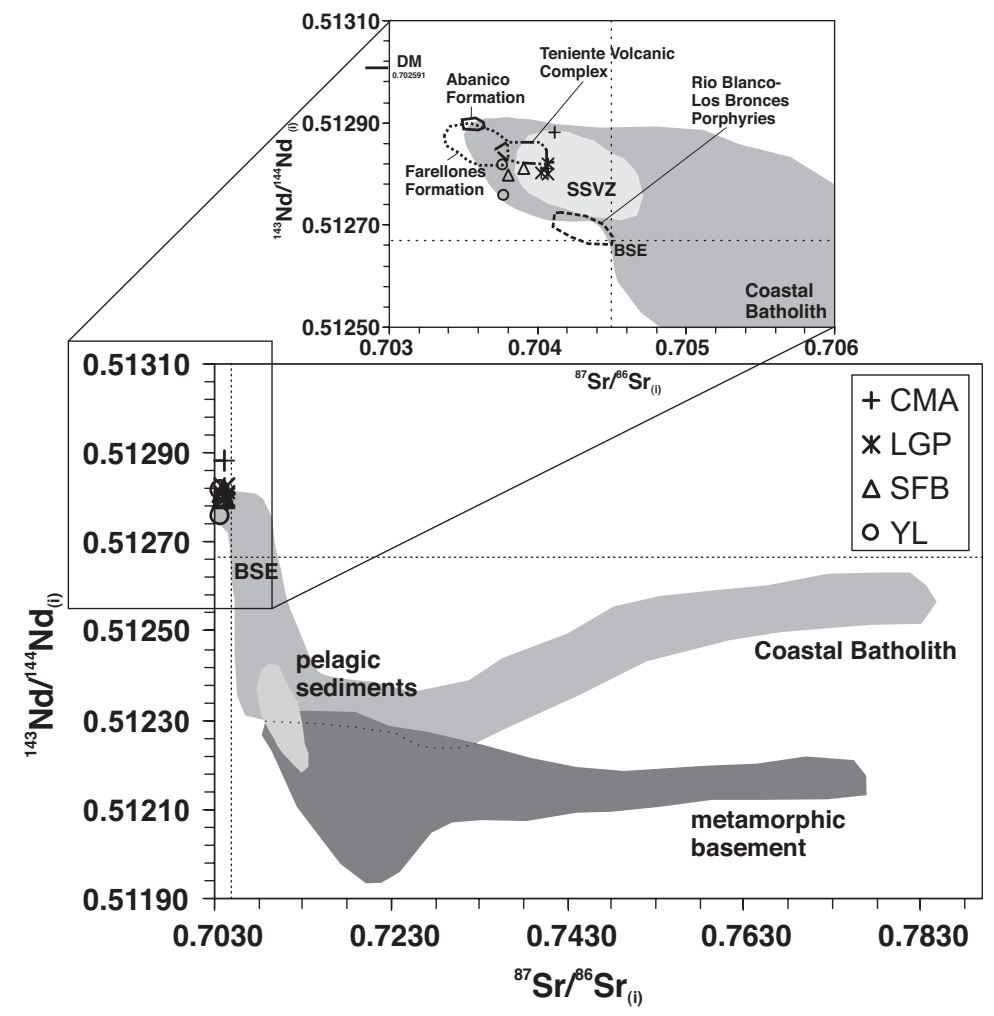

FIG. 9. Initial isotope diagrams for ${ }^{87} \mathrm{Sr} /{ }^{86} \mathrm{Sr}$ versus ${ }^{143} \mathrm{Nd} /{ }^{144} \mathrm{Nd}$ with fields of southern Central Andes metamorphic basement rocks (Lucassen et al., 2004), central Chilean Coastal Batholith rocks (Parada et al., 1999), Pacific pelagic sediments (Othmann et al., 1989), Teniente Volcanic Complex (Kay et al., 2005), Río Blanco-Los Bronces porphyries (Stern and Skewes, 1995), Farellones and Abanico formations (Nyström et al., 2003), southernmost Southern Volcanic Zone (SSVZ) (Kilian and Behrmann, 2003) and DM (Michard et al., 1985; McCulloch and Black, 1984). 


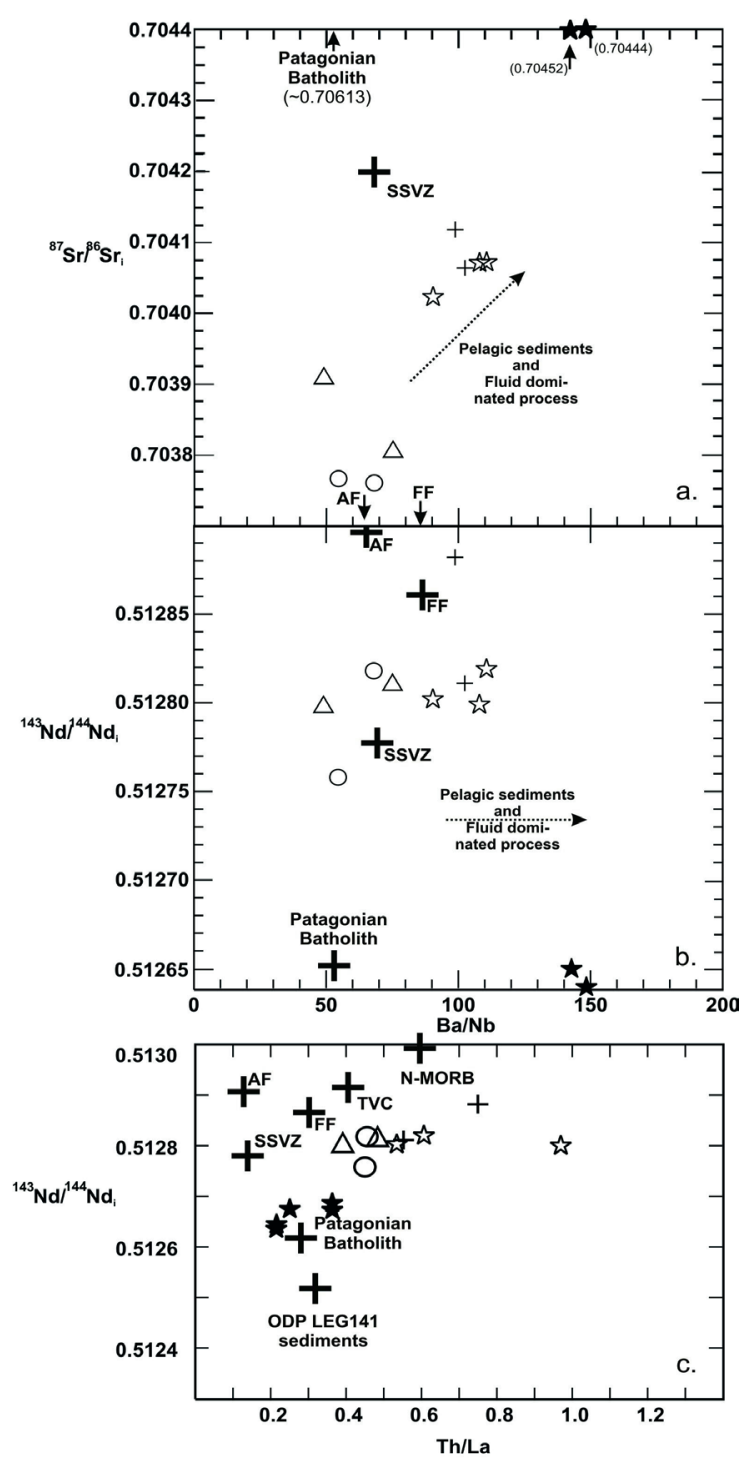

FIG. 10. a. $\mathrm{Ba} / \mathrm{Nb}$ versus ${ }^{87} \mathrm{Sr} /{ }^{86} \mathrm{Sr}_{i} ;$ b. $\mathrm{Ba} / \mathrm{Nb}$ versus ${ }^{143} \mathrm{Nd} /{ }^{144} \mathrm{Nd}_{i}$ and c. Th/La versus ${ }^{143} \mathrm{Nd} /{ }^{144} \mathrm{Nd}_{i}$. Average values of Patagonian Batholith, southernmost Southern Volcanic Zone (SSVZ), and sediments after Pankhurst et al. (1999), López-Escobar et al. (1993) and Kilian and Behrmann (1997), Teniente Volcanic Complex (TVC) (Kay et al., 2005), Río Blanco-Los Bronces porphyries (Stern and Skewes, 1995). Farellones (FF) and Abanico formations (AF) (Nyström et al., 2003) and, Kilian and Behrmann (2003), respectively. Symbols as in figure 6 .

significantly higher $\mathrm{Th} / \mathrm{La}$ ratios. Whereas higher $\mathrm{Th} /$ La ratios might account for recycling subarc mantle lithosphere or continental crust into the mantle source, lower $\mathrm{Th} / \mathrm{La}$ ratios of the new data and characteristic for Río Blanco-Los Bronces porphyries indicate recycling of oceanic crust through the subduction zone into a mantle source (Kerrich et al., 1999).
Initial $\varepsilon H f$-isotope data plotted against $\varepsilon \mathrm{Nd}_{\mathrm{i}}$ ratios (Fig. 11a) separate one sample (FA-1) of the Cerro Mesón Alto massif from the rest of all plutons. The more radiogenic $\varepsilon \mathrm{Nd}_{i}$ value of the Cerro Mesón Alto massif (FA-1) is not accompanied by a higher $\varepsilon \mathrm{Hf}_{\mathrm{i}}$ value. This result can be considered to represent an outlier since major and trace elements do not indicate any unusual behaviour compared to the other Cerro Meson Alto sample. Plotting ${ }^{176} \mathrm{Hf} /{ }^{177} \mathrm{Hf}_{\mathrm{i}}$ versus ${ }^{87} \mathrm{Sr} /{ }^{86} \mathrm{Sr}_{\mathrm{i}}$ isotopic data a split between the Cerro Mesón Alto and La Gloria pluton group (about 10-11 Ma) and the San Francisco Batholith and Yerba Loca stock rocks (about $14.8 \mathrm{Ma}$ ) becomes evident, with the first group being slightly more radiogenic in Sr isotopes (Fig. 11b). Variations in initial isotopic composition are still less in $\mathrm{Nd}$ and smallest in the case of Hf. This observation underlines the fact that Hf isotope systematics represents the most robust system in subduction weakly or not affected by dehydration. It possibly represents original or residual mantle source values. Figure 11c shows whole rock and zircon initial Hf-isotope ratios versus age indicating that all samples plot above the CHUR and below the DM evolution lines. This observation indicates a residual depleted mantle involved at an early stage of magma evolution. 'Mantle'- $\mathrm{T}_{\mathrm{DM}}$ ages calculated for the Hf whole rock data from the Cerro Mesón Alto and La Gloria pluton granitoids, however, lie in the future (Table $3 \mathrm{a}-\mathrm{b}$ ). Since ${ }^{176} \mathrm{Lu} /{ }^{177} \mathrm{Hf}$ ratios are high and samples are young, as confirmed by geochemical data, they most probably did not derive directly from a depleted mantle source. An estimated 'crustal' $\mathrm{T}_{\mathrm{DM}}$ (two-stage model) with a ${ }^{176} \mathrm{Lu} /{ }^{177} \mathrm{Hf}$ ratio $=0.015$ (average continental crust; bulk silicate earth, Goodge and Vervoort, 2006) should give a more realistic age of separation of the host magma source from depleted mantle (DM) or crustal forming events. The widest whole rock crustal- $\mathrm{T}_{\mathrm{DM}}$ age range is given by the Yerba Loca stock (510-590 Ma), whereas the San Francisco Batholith lies between 520 and 530 Ma. Both, the Cerro Mesón Alto massif and the La Gloria pluton values range between 550 and $570 \mathrm{Ma}$. Figure 11c shows that whole rock and zircon single grain initial ${ }^{176} \mathrm{Hf} /{ }^{177} \mathrm{Hf}$ isotopic data are principally overlapping except for the $c a$. 14.8 Ma zircon samples from the Yerba Loca stock and San Francisco Batholith (zircon: 110-540 Ma; WR: 510-590 Ma) with initial Hf-ratios slightly more radiogenic than the entire whole rock and zircon mineral data set. 

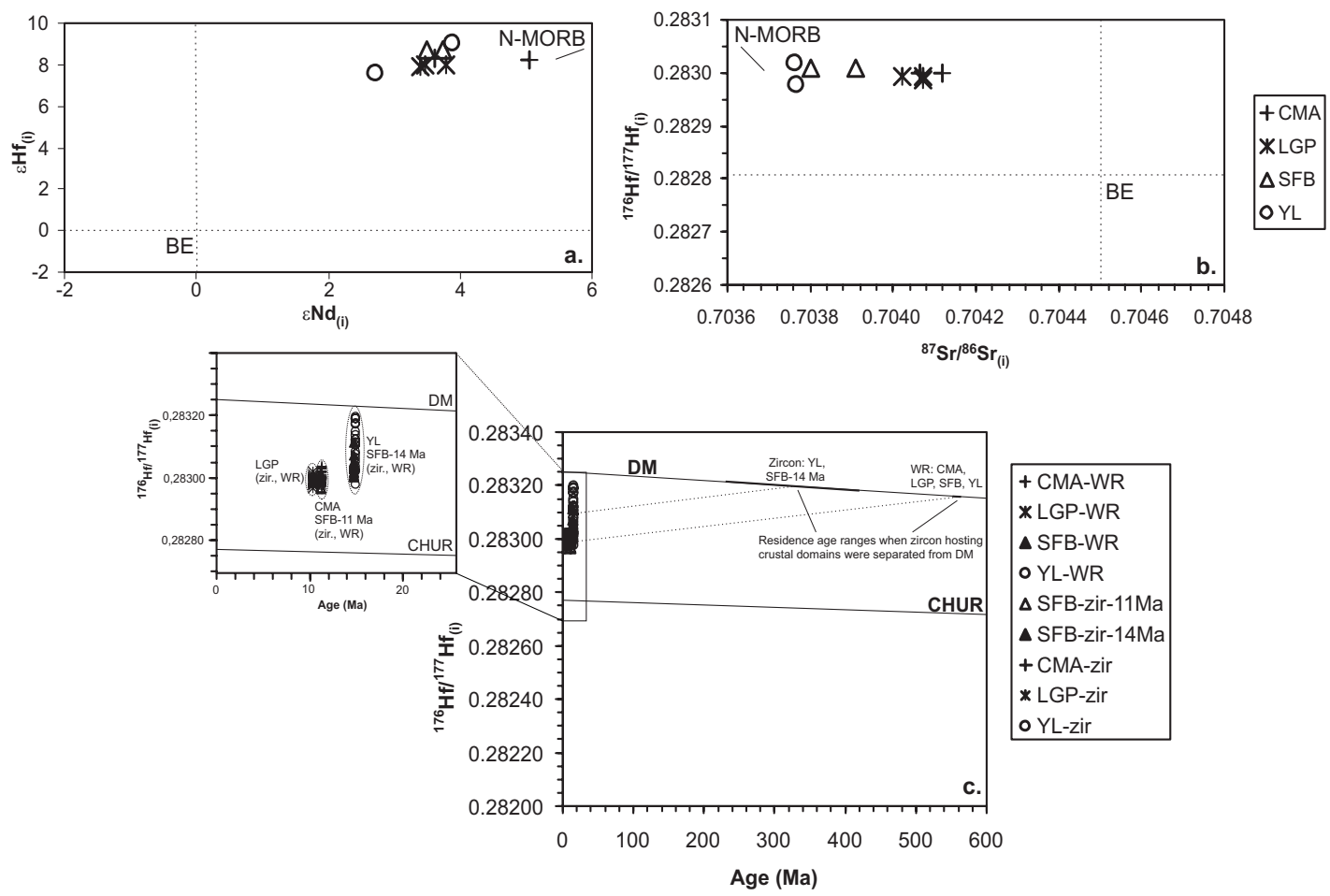

FIG. 11. Initial Hf-isotope plots, a. whole rock $\varepsilon N d$ versus $\varepsilon \mathrm{Hf}$; b. whole rock ${ }^{87} \mathrm{Sr} /{ }^{86} \mathrm{Sr}$ versus ${ }^{176} \mathrm{Hf} /{ }^{177} \mathrm{Hf}$ and, c. age versus whole rock and zircon single grain ${ }^{176} \mathrm{Hf} /{ }^{177} \mathrm{Hf}$ data; CHUR parameters of Blichert-Toft and Albarede (1997).

\section{Middle-to-Late Miocene source rock}

In a subduction environment, element enrichment is often explained through either varying amounts of essentially lower crustal components, metasomatic fluid circulation from the subducting ocean plate, dehydration of subducted sediments, or partial melting of sediments. Adakitic geochemical rock signatures (high $\mathrm{Sr} / \mathrm{Y}(>40), \mathrm{La} / \mathrm{Yb}(\mathrm{n})$ subscript $(>20)$ ratios; low high field strength element concentrations; depleted $(<0.704){ }^{87} \mathrm{Sr} /{ }^{86} \mathrm{Sr}$ ratios (Defant and Drummond, 1990)) have been described as relatively common in the Neogene of the central Andes, but can be excluded for the granitoids herein presented.

The ages of all samples versus $\mathrm{Sr} / \mathrm{Y}$ ratios (Fig. 12) indicate a slight-to-no tendency to increasing $\mathrm{Sr} / \mathrm{Y}$ ratios with decreasing ages in the temporal interval from 20-5.5 Ma. It is notable that there is a higher $\mathrm{Sr} / \mathrm{Y}$ ratio (88 and 113) detected for the two dacite porphyry samples (Stern and Skewes, 1995) from the Río Blanco-Los Bronces deposit as observed by several authors (e.g., Thieblemont et al., 1997) who suggested that adakitic magmas $(\mathrm{Sr} / \mathrm{Y}>40)$ are involved in giant porphyry copper deposits. Furthermore, La Gloria pluton and San Gabriel granitoids are barren, yet plot on the limit or inside the adakite field.

An arc-like signature generally characterizes the Middle to Late Miocene rocks. $\mathrm{Ba} / \mathrm{Th}, \mathrm{Ta} / \mathrm{Yb}$ and $\mathrm{Th} / \mathrm{Yb}$ ratios indicate for all plutonic rocks an enriched mantle influence, which might be explained through a slight subduction component in the source area and /or crustal contamination during magma ascent. Higher $\mathrm{Ba} / \mathrm{La}$ ratios show a tendency to subduction fluids as enrichment factor (La Gloria pluton and the older La Obra pluton), whereas higher $\mathrm{La} / \mathrm{Yb}$ might suggest terrigeneous sediment influence (Cerro Mesón Alto). The remaining samples (e.g., Río Blanco-Los Bronces porphyries) plot intermediate to these trends and might indicate both processes for the enrichment of especially large ion lithophile elements (LILE).

The available isotopic compositions are compatible with two distinct mantle sources for two distinct rock and age groups: the 10-11 Ma group and the older, 14.8 Ma group (Fig. 9). All samples plot in the mantle array between N-MORB and Bulk Silicate Earth compositions. Whereas the first group shows 


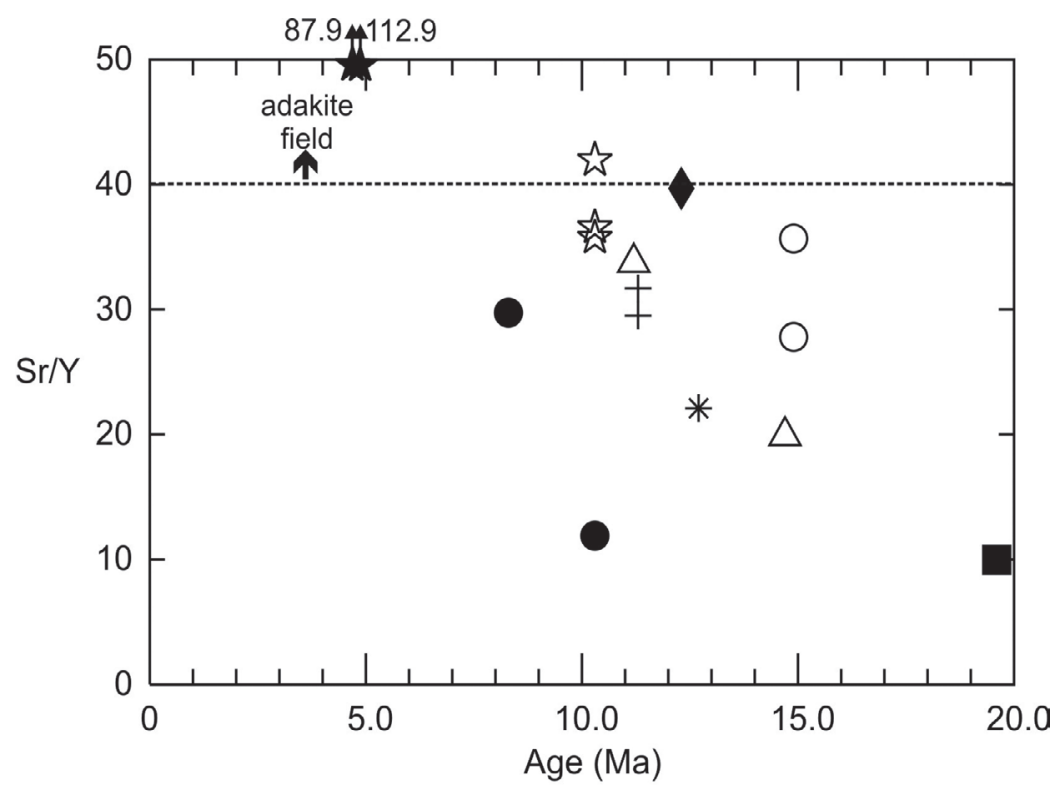

FIG. 12. Age versus $\mathrm{Sr} / \mathrm{Y}$ for new data and selected rocks from Kay et al. (2005) and Río Blanco-Los Bronces rocks (Stern and Skewes, 1995). The adakite field is taken after Defant and Drummond (1990). Symbols as in figure 6 . slightly more enriched Sr-isotope characteristics, Hf isotopic signatures are relatively uniform in both data sets. A major difference is shown by ${ }^{187} \mathrm{Os} /{ }^{186} \mathrm{Os}_{(i)}$ isotopic data from magmatic magnetite minerals of each of the four plutons. Samples from the first group (CMA, LGP) are clearly characterized by continental crust input (average ${ }^{187} \mathrm{Os} /{ }^{188} \mathrm{Os}_{(\mathrm{i})}=0.33$; $\varepsilon \mathrm{Os}_{(\mathrm{i})}=+151$ and $+168 \%$; Table 5) whereas the second group (YL, SFB) indicates with its slightly positive $\varepsilon \mathrm{Os}_{(\mathrm{i})}$ values (average ${ }^{187} \mathrm{Os} /{ }^{188} \mathrm{Os}_{(\mathrm{i})}=0.17$; $\varepsilon \mathrm{Os}_{(\mathrm{i})}=+28$ and $+37 \%$; Table 5) that the magma source was enriched in Re, presumably being the result of ocean floor sediments recycled into the source area or by the way of crustal contamination during magma ascent. These isotope characteristics agree with the trace element ratios (e.g., Ta/Hf, Ta/ $\mathrm{Yb}, \mathrm{Th} / \mathrm{Yb}$ ) characteristic of the intrusive bodies, as presented above. Increased $\mathrm{Ba} / \mathrm{Nb}$ ratios might suggest preferential trace element enrichment by fluid processes or pelagic sediments or, when combined with increasing initial ${ }^{87} \mathrm{Sr} /{ }^{86} \mathrm{Sr}$ ratios, to crustal input. Variation in $\mathrm{Th} / \mathrm{La}$ ratios with relatively constant radiogenic initial $\mathrm{Nd}$ isotopic values imply slight crustal or ocean-floor sediment input for nearly all samples except one La Gloria pluton (FA-5) and one Cerro Mesón Alto (FA-1) sample. Their increased $\mathrm{Th} /$ La might reflect high temperature mobilization enrichment of Th compared to La.

Magmatic evolution of the Middle-to-Late Miocene intrusions may thus be explained either by a slightly enriched mantle source or by a two- stage model. The two-stage model projects the emplacement composition back to a hypothetical crustal formation/mantle depletion event, assuming a crustal ${ }^{176} \mathrm{Lu} /{ }^{177} \mathrm{Hf}$ of 0.015 ; and yields a minimum age for the hypothetical crustal reservoir.

Such model ages range between 470 and $630 \mathrm{Ma}$ (Early Ordovician to Neoproterozoic) for sample FA-3 from the Cerro Mesón Alto massif and a shorter age range of 510 to $620 \mathrm{Ma}$ (Early Cambrian to Neoproterozoic) for both FA-4 from the La Gloria pluton and FA-7 from the younger San Francisco Batholith sample. A more significant variation in ${ }^{176} \mathrm{Hf} /{ }^{177} \mathrm{Hf}$ ratios is seen in zircon samples from Yerba Loca and the older sample (14.7 Ma) from the San Francisco Batholith. Variable model crustal residence $\mathrm{T}_{\mathrm{DM}}$ ages are recognised: 300-540 Ma (FA-8, San Francisco Batholith), 160 to $490 \mathrm{Ma}$ (FA-9, Yerba Loca stock) and 110 to $360 \mathrm{Ma}$ (FA11, YL). This distinct isotopic data set for the two age groups (about $11 \mathrm{Ma}$ and $14.8 \mathrm{Ma}$ ) suggests either a temporal change in a single magma source composition by different portions of contaminants, or the involvement of at least two magma sources or distinct differentiation processes.

Whole rock crustal Hf- $\mathrm{T}_{\mathrm{DM}}$ ages from Neoproterozoic to Early Cambrian show the same crustal $\mathrm{Hf}-\mathrm{T}_{\mathrm{DM}}$ age range as zircon single grain ages for the Cerro Mesón Alto massif and La Gloria pluton, being slightly less pronounced for the San Francisco Batholith. The oldest San Francisco Batholith rock and Yerba Loca stock are exceptional in that they yield 


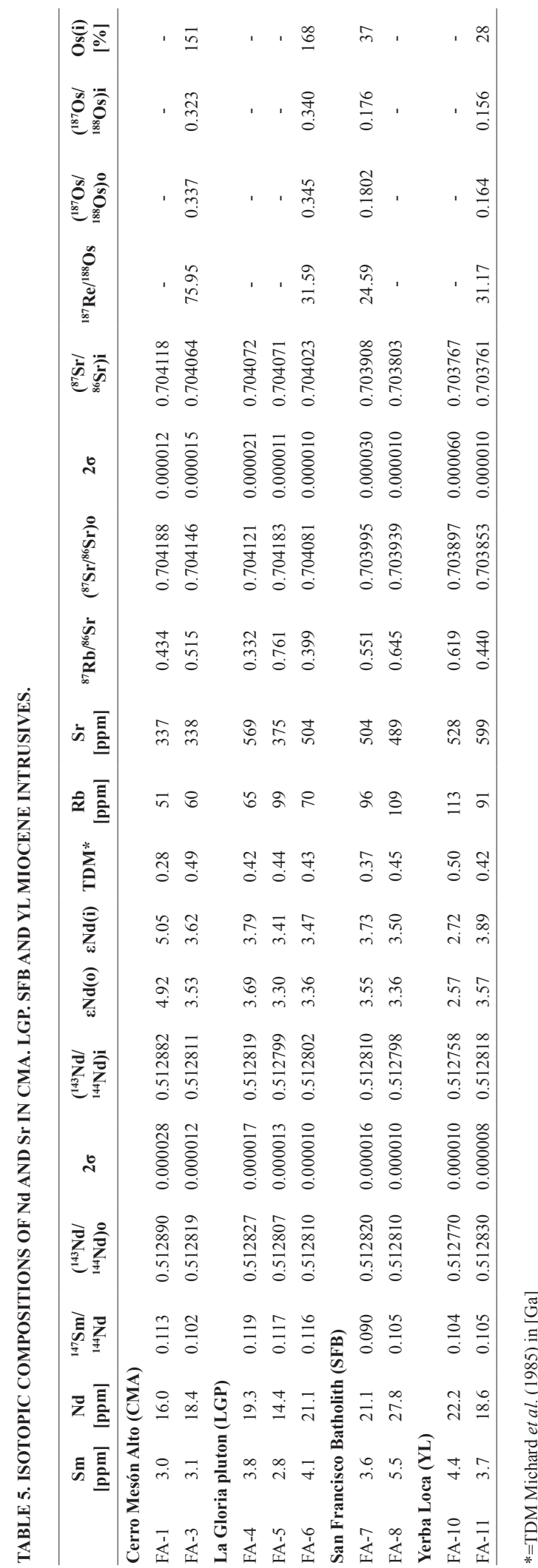

the same crustal Hf- $\mathrm{T}_{\mathrm{DM}}$ ages for whole rock analyses but a range from $540 \mathrm{Ma}$ to $110 \mathrm{Ma}$ (Cambrian to Early Cretaceous) for zircon single grains. This rather identical Neoproterozoic to Early Cambrian crustal $\mathrm{Hf}_{\mathrm{DM}}$ age for zircon and whole rock from the younger plutons suggests that zircon grains originated from the same magma as the zircon-bearing intrusives and, therefore, the modelled crustal residence age may be used as an estimate for a crustal-type magma separation from a residual depleted mantle source. Hf isotopes from the older zircon samples suggest contribution from a more juvenile mantle residual. These results do not agree with the $2 \mathrm{Ga} H f-m o d e l$ ages and their interpretation presented in Montecinos et al. (2008) on coeval (15-10 Ma) intrusions at $33^{\circ} \mathrm{S}$. They described their obtained model ages as consistent with Sm-Nd model and upper intercept $\mathrm{U}-\mathrm{Pb}$ ages from zircons of distinct Proterozoic and Phanerozoic lithologies (1.9$2.1 \mathrm{Ga}$ ) in the central Andes between $21^{\circ} \mathrm{S}$ and $32^{\circ} \mathrm{S}$ and $36^{\circ}$ and $41^{\circ} \mathrm{S}$ (in Franz et al., 2006). Consequently, this inconsistency outlines a distinctive petrogenetic evolution for the herein studied $33^{\circ} \mathrm{S}$ to $34^{\circ} \mathrm{S}$ plutons compared to the one proposed in Montecinos et al. (2008) using the much older lithologies reported by Franz et al. (2006).

Inheritance in zircon single grains in all intrusives bodies is not characteristic, as has been recognized in mineralized porphyric intrusions (6.3-5.0 Ma) of the Río Blanco-Los Bronces Cu (-Mo) deposit (Deckart et al., 2005), where zircon core ages range mainly between the Carboniferous and Mesoproterozoic. Furthermore, zircon grains from andesites of the volcanic Farellones Formation ( $c a .17 \mathrm{Ma}$ ) cropping out in the Río Blanco mine sector yield one Neoproterozoic age for its upper discordia intercept (Deckart et al., 2005), whereas U-Pb SHRIMP dating of a Miocene ignimbrite in the El Teniente area indicated abundant Oligocene ages together with a few $114 \mathrm{Ma}$ and $268 \mathrm{Ma}$ ages (Godoy et al., in press). Zentilli et al. (2001) described as a common phenomenon xenocrystic zircons in older Cretaceous rocks of the Punta del Cobre $\mathrm{Cu}-\mathrm{Fe}(\mathrm{Au})$ District and in the El Soldado $\mathrm{Cu}$ District of northern and central Chile, respectively. They suggested that during ascent felsic magmas associated with the mineralization picked up zircons from the Coastal Cordilleran granitic basement. However, zircon results in this study do not confirm this type of contamination process for our plutonic setting, which points to a different magma generation and emplacement process for the 15-10 Ma barren intrusions compared to the fertile 
plutonic younger and older pulses in the study area and further northeast in the Coastal Cordillera area, respectively. Instead, assimilation seems to have taken place on a macroscopic scale. Mafic enclaves (mainly composed of hornblende, plagioclase and quartz minerals) are characteristic in both the Cerro Mesón Alto massif and La Gloria pluton. It is important to point out that these mafic enclaves are completely absent from mineralization-associated rocks in the Río Blanco mine area (e.g., San Francisco Batholith, Yerba Loca stock). The homogeneity and occurrence of the entire isotope range in the mantle array of the depleted quadrant supports, on the other hand, the suggestion that crustal components must have been of rather minor importance in the generation of these rocks.

Geochemical and isotope characteristics of subduction zone-related plutonic bodies in the central Andes between $33^{\circ}$ and $34^{\circ} \mathrm{S}$ indicate an enriched subarc mantle source which was preferentially enriched in LIL and some HFS elements. It is suggested that element enrichment occurred either through dehydration fluids from subducted sediments which replenished the subarc mantle source (YL, SFB), or/and through partial melting or incorporation of a small amount of fragments of crustal material without homogenisation within the ascending magma (CMA, LPG). Finally, source enrichment processes do not appear to have been homogenous, and can be discarded as a process responsible for the separation of the younger age group (10-11 Ma), Cerro Mesón Alto massif, La Gloria pluton and the younger San Francisco Batholith from the older (14.8 Ma) San Francisco Batholith and Yerba Loca stock rocks.

\section{Conclusions}

Barren plutons occur between two supergiant porphyry $\mathrm{Cu}(-\mathrm{Mo})$ deposits in the Central Andes, Chile, between $33^{\circ}$ and $34^{\circ} \mathrm{S}$. Four of them (Cerro

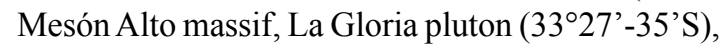
Yerba Loca stock and San Francisco Batholith) have U-Pb LA-ICPMS emplacement ages ranging from 10.3 to $15 \mathrm{Ma}$. In the study area magmatism does not reflect the general eastward arc front migration postulated by e.g., Kurtz et al. (1997) and Kay et al. $(1999,2005)$. The Cerro Mesón Alto intrusive is slightly older than the La Gloria pluton but coeval with one age obtained from the more westerly located San Francisco Batholith. The magmatic arc younging hypothesis is applicable on a regional scale (e.g., from the coast to the Principal Cordillera $\mathrm{W}$ to $\mathrm{E}$ transect) but not on a local scale $\left(70^{\circ} 30^{\prime}-69^{\circ} 45^{\prime}\right)$. It, however, does not take into account either displacement by covered sinistral transfer faults, a complex tectonic control of plutonic emplacement or even younger intrusive events at about $1.1 \mathrm{Ma}$, as described for the Volcán valley by Godoy (1998) and Baeza (1999), mixed in the general Miocene-Pliocene magmatism of the study area. The latter observation points to a stationary (12-1 Ma) magmatic arc at these latitudes.

A quartz monzodioritic to quartz monzogabbroic composition and a metaluminous character characterize the plutonic complexes.

Rare earth element patterns suggest that amphibole and garnet were possibly minor components on the Late Miocene magma source in contrast with the Neogene adakites and the Río Blanco-Los Bronces porphyries. The $(\mathrm{La} / \mathrm{Yb})_{\mathrm{n}}$ ratios indicate an only slightly higher fractionation degree for the southern plutons compared to the northern ones. Río Blanco-Los Bronces porphyries indicate with their elevated ratio most probably residual garnet in the source. The adakite -like geochemical features outlined for Late Miocene porphyritic intrusions at Los Pelambres located ca. $100 \mathrm{~km}$ north of the study area (Reich et al., 2003) could not be supported by the coeval barren intrusions analyzed herein. When the Middle-to-Late Miocene plutonic rocks studied here are compared with their volcanic Miocene Farellones Formation host rocks, a direct genetic relation, as mentioned for the Early-to-Middle Miocene intrusion and the Eocene-Early Miocene Abanico Formation (cf. López-Escobar et al., 1979; Kurtz et al., 1997), could not be confirmed. Yet, an arclike signature is suggested for all rocks, including volcanics and mineralized rocks. Trace element ratios for all plutonic rocks indicate an enriched mantle influence with possibly a slight subduction component and/or crustal contamination during magma generation.

Isotopic ratios for the plutons also indicate a slightly enriched mantle source with only small isotopic variations, which might be accounted for by a slightly heterogeneous source. Nd-Sr isotopic ratios show small variations, whereas Hf-Sr isotopic data separate the southern from the northern intrusions, with higher radiogenic $\mathrm{Sr}$ for the southern ones. Crustal Hf- $\mathrm{T}_{\mathrm{DM}}$ modelling indicates a magma source that separated from the depleted mantle residual around 550-500 Ma and evolved until 11-10 Ma ago when magma re-melted and was emplaced at crustal levels 
as indicated by both whole rock (entire data set) and single zircon Hf-isotope (CMA, LGP, SFB) data. The older group (14.8 Ma; SFB, YL) is characterized by similar Hf-isotopic whole rock data but shows a wide range of zircon Hf-data suggesting contributions from a more juvenile mantle residual. Magma ascent has been direct for partly coeval volcanics but shows slight LIL element enrichment for the entire middle to Late Miocene plutonic magmatism.

The data presented here supports a slightly distinctive magma evolution through time from older to barren younger magmatism, different from the one recognised in the later, fertile one. Further studies on barren intrusives as well as a larger geochemical data set on the fertile magma are necessary. Some features that need more investigation and may relate to differences in the magmatic evolution of barren to mineralized intrusives are: the barren plutons contain mafic enclaves and show no zircon inheritance, mineralization-associated or -hosting intrusion show generally no mafic enclaves and no zircon inheritance, whereas the mineralized intrusives lack mafic enclaves and contain inherited zircon grains.

\section{Acknowledgements}

This study was financed through the Fundación Andes Project No. C-13960/32. Geologists M. Farías and A. Fock are thanked for assistance during field trips and $\mathrm{J}$. Vargas for sample preparations in the facility of the Geology Department, University of Chile. We would like to thank N. Pearson and W. Griffin for supplying analytical work at GEMOC, Australia and F. Barra for the Os data on magnetite at the University of Arizona, USA. We appreciate the constructive comments of S.M. Kay and J.-P. Liégeois on a prior version of this manuscript. The authors acknowledge the critical review by S.M. Kay, R. Pankhurst and D. Morata. J. Le Roux is friendly thanked for having checked the English of this paper. This research used instrumentation funded by ARC LIEF and DEST Systemic Infrastructure Grants, Macquarie University and Industry. This is the contribution No. 618 from the ARC National Key Centre for Geochemical Evolution and Metallogeny of Continents. We acknow-ledge willingness from FONDECYT project 1095099 to include our results into its modelling.

\section{References}

Baeza, O. 1999. Análisis de litofacies, evolución depositacional y análisis estructural de la Formación Abanico en el área comprendida entre los ríos Yeso y Volcán, Región Metropolitana. Memoria de Título (Inédito), Universidad de Chile, Departamento de Geología: 119 p.

Black, L.P.; Gulson, B.L. 1978. The age of the Mud Tank carbonatite, Strangways Range, Northern Territory. BMR Journal of Australian Geology and Geophysics 3: 227-232.

Blichert-Toft, J.;Albarède, F. 1997. The Lu-Hfgeochemistry of chondrites and the evolution of the mantle-crust system. Earth and Planetary Science Letters 148: 243-258. Erratum. Earth and Planetary Science Letters 154 (1998): 349 p.

Borg, L.E.; Clynne, M.A.; Bullen, T. 1997. The variable role of slab-derived fluids in the generation of a suite of primitive calc-alkaline lavas from the southernmost Cascades, California. The Canadian Mineralogist 35: 425-452.

Creaser, R.A.; Papanastassiou, D.A.; Wasserburg, G.J. 1991. Negative thermal ion mass spectrometer of Os, Re and Ir. Geochimica et Cosmochimica, Acta 55: 397-401.

Cornejo, P.; Mahood, G. 1997. Seeing past the effects of re-equilibration to reconstruct magmatic gradients in plutons: La Gloria Pluton, central Chilean Andes. Contribution to Mineralogy and Petrology 127: 159-175.

Davidson, J.; Mpodozis, C. 1991. Regional geologic setting of epithermal gold deposits, Chile. Economic Geology 86: 1174-1186.

De Bievre, P.; Taylor, P.D.P. 1993. Table of the isotopic composition of the elements. International Journal of Mass Spectrometry and Ion Processes 123: $149 \mathrm{p}$.

Deckart, K.; Clark, A.H.; Aguilar, C.; Vargas, R. 2005. Magmatic and hydrothermal chronology of the supergiant Río Blanco Porphyry Copper Deposit, Central Chile: Implications of an integrated $\mathrm{U} / \mathrm{Pb}$ and ${ }^{40} \mathrm{Ar} /{ }^{39} \mathrm{Ar}$ database. Economic Geology 100 (5): 905-934.

Defant, M.J.; Drummond, M.S. 1990. Derivation of some modern arc magmas by melting of young subducted lithosphere. Nature 347: 662-665.

Drummond, M.S.; Defant, M.J.; Kepezhinskas, P.K. 1996. Petrogenesis of slab-derived trondhjemitetonalite-dacite/adakite magmas. Transactions of the Royal Society of Edinburgh: Earth Sciences 87: 205-215.

Elliot, T. 2003. Tracers of the slab. In Inside the subduction factory (Eiler, J.; editor). American Geophysical Union Geophysical Monograph 138: 23-45.

Ewart, A.; Collerson, K.D.; Regelous, M.; Wendt, J.I.; Niu, Y. 1998. Geochemical evolution within the 
Tonga-Kermadec-Lau arc-backarc system: the role of varying mantle wedge composition in space and time. Journal of Petrology 39: 331-368.

Fock, A. 2005. Cronología y tectónica de la exhumación en el Neógeno de los Andes de Chile Central entre los $33^{\circ}$ y los $34^{\circ} \mathrm{S}$. Memoria de Título (Inédito), Universidad de Chile, Departamento de Geología: 179 p.

Franz, G.; Lucassen, F.; Kramer, W.; Trumbull, R.B.; Romer, R.L.; Wilke, H.-G.; Viramonte, J.G.; Becchio, R.; Siebel, W. 2006. Crustal Evolution at the Central Andean Continental Margin: a Geochemical Record of Crustal Growth, Recycling and Destruction (Oncken, O.; Chong, G.; Franz, G.; Giese, P.; Götze, H.-J.; Ramos, V.; Strecker, M.R.; Wigger, P.; editors). The Andes-Active Subduction Orogeny. Springer, Chapter 3: 45-64.

Godoy, E. 1998 Intrusivos sintectónicos entre los ríos Aconcagua y Cachapoal, Andes de Chile Central. In Congreso Latinoamericano de Geología, No. 10, Actas 2: 149-154. Buenos Aires.

Godoy, E. 2005. Comentario sobre 'High magma oxidation state and bulk crustal shortening: key factors in the genesis of Andean porphyry copper deposits, Chile (31-34 $\left.{ }^{\circ} \mathrm{S}\right)$. Revista Geológica de Chile 32 (1): 155-158.

Godoy, E.; Schilling, M.; Solari, M.; Fock, A. En prensa. Geología del Área Rancagua-San Vicente de Tagua Tagua. Servicio Nacional de Geología y Minería, Carta Geológica de Chile, Serie Geología Básica, No. 118, 1 mapa escala 1:100.000.

Goodge, J.W.; Vervoort, J.D. 2006. Origin of Mesoproterozoic A-type granites in Laurentia: Hf isotope evidence. Earth and Planetary Science Letters 243: 711-731.

Griffin, W.L.; Pearson, N.J.; Belousova, E.; Jackson, S.E.; O'Reilly, S.Y.; van Achterberg, E.; Shee, S.R. 2000. The Hf isotope composition of cratonic mantle: LAM-MC-ICPMS analysis of zircon megacrysts in kimberlites. Geochimica and Cosmochimica, Acta 64: 133-147.

Hickey, R.L.; Frey, F.A.; Gerlach, D.C.; López-Escobar, L. 1986. Multiple source for basaltic arc rocks from the southern volcanic zone of the Andes (34-41 $\left.{ }^{\circ} \mathrm{S}\right)$ : Trace element and isotopic evidence for the contributions from subducted oceanic crust, mantle and continental crust. Journal of Geophysical Research 91: 5963-5983.

Jackson, S.E.; Pearson, N.J.; Griffin, W.L.; Belousova, E.A. 2004. The application of laser ablationinductively coupled plasma-mass spectrometry to in situ $\mathrm{U} / \mathrm{Pb}$ zircon geochronology. Chemical Geology 211: 47-69.

Kay, S.M.; Mpodozis, C. 2001. Central Andean ore deposits linked to evolving shallow subduction systems and thickening crust. Geological Society of America (GSA) Today 11 (3): 4-9.

Kay, S.M.; Godoy, E.; Kurtz, A. 2005. Episodic arc migration, crustal thickening, subduction erosion, and magmatism in the south-central Andes. The Geological Society of America Bulletin 117 (1-2): 67-88.

Kay, S.M.; Mpodozis, C.; Coira, B. 1999. Neogene magmatism, Tectonism, and Mineral Deposits of the Central Andes ( $22^{\circ}$ to $33^{\circ} \mathrm{S}$ Latitude). In Geology and ore deposits of the central Andes (Skinner, B.; editor). Society of Economic Geology, Special Publication 7: 27-59.

Kay, S.M.; Kurtz, A.; Godoy, E. 1995. Tertiary magmatic and tectonic framework of the El Teniente copper deposit, southern Chile $\left(34^{\circ} \mathrm{S}-35^{\circ} \mathrm{S}\right)$. Geological Society of America, Abstracts with Programs 27 (6): p. 409.

Kerrich, R.; Wyman, D.; Hollings, P.; Polat, A. 1999. Variability of $\mathrm{Nb} / \mathrm{U}$ and $\mathrm{Th} / \mathrm{La}$ in 3.0 to $2.7 \mathrm{Ga} \mathrm{Su}-$ perior Province ocean plateau basalts: implications for the timing of continental growth and lithosphere recycling. Earth and Planetary Science Letters 168 (1): 101-115.

Kilian, R.; Behrmann, J.H. 1997. Southern Chile trench sediments: Chemical and isotopic constraints on their source. In Congreso Geológico Chileno, No. 8, Actas 3: 1661-1665. Antofagasta.

Kilian, R.; Behrmann, J.H. 2003. Geochemical constraints on the source of Southern Chile trench sediments and their recycling in arc magmas of the Southern Andes. Journal of the Geological Society 160: 57-70.

Kurtz, A.C.; Kay, S.M.; Charrier, R.; Farrar, E. 1997. Geochronology of Miocene plutons and exhumation history of the El Teniente region, Central Chile $\left(34-35^{\circ} \mathrm{S}\right)$. Revista Geológica de Chile 24 (1): 75-90.

López-Escobar, L.; Kilian, R.; Kempton, P.D.; Tagiri, M. 1993. Petrology and geochemistry of Quarternary rocks from the Southern Volcanic Zone of the Andes between $41^{\circ} 30^{\prime}$ and $46^{\circ} 00^{\prime} S$, Chile. Revista Geológica de Chile 20 (1): 33-56.

López-Escobar, L.; Frey, F.A.; Oyarzún, J. 1979. Geochemical characteristics of Central Chile $\left(33^{\circ}-34^{\circ} \mathrm{S}\right)$ granitoids. Contributions to Mineralogy and Petrology 113 (3): 381-393. 
Lucassen, F.; Trumbull, R.; Franz, G.; Creixell, C.; Vásquez, P.; Romer, R.L.; Figueroa, O. 2004. Distinguishing crustal recycling and juvenile additions at active continental margins: the Paleozoic to recent compositional evolution of the Chilean Pacific margin (36-41 $\left.{ }^{\circ} \mathrm{S}\right)$. Journal of South American Earth Sciences 17: 103-119.

Michard, A.; Gurriet, P.; Soudant, M.; Albarede, F. 1985. $\mathrm{Nd}$ isotopes in French phanerozoic shales: external versus internal aspects of crustal evolution. Geochimica et Cosmochimca Acta 49: 601-610.

McCulloch, M.T.; Black, L.P. 1984. Sm-Nd isotopic characteristics of Enderby Land granulites and evidence for the redistribution of Sm and Nd during metamorphism. Earth and Planetary Science Letters 71: 46-58.

Montecinos, P.; Schärer, U.; Vergara, M.; Aguirre, L. 2008. Lithospheric origin of Oligocene-Miocene magmatism in Central Andes: $\mathrm{U}-\mathrm{Pb}$ ages and $\mathrm{Sr}-$ $\mathrm{Pb}$-Hf isotope composition of minerals. Journal of Petrology 49 (3): 555-580.

Nägler, T.F.; Frei, R. 1997. Plug in plug osmium distillation. Schweizer Mineralogische und Petrographische Mitteilungen 77: 123-127.

Niu, Y.; O'Hara, M.J. 2003. Origin of ocean island basalts: A new perspective from petrology, geochemistry and mineral physics considerations. Journal of Geophysical Research: Solid Earth 108 (B4): 2209.

Nowell, G.M.; Kempton, P.D.; Noble, S.R.; Fitton, J.G.; Saunders, A.D.; Mahoney, J.J.; Taylor, R.N. 1998. High precision Hf isotope measurements of MORB and OIB by thermal ionization mass spectrometry: Insights into the depleted mantle. Chemical Geology 149: 211-233.

Nyström, J.O.; Vergara, M.; Morata, D.; Levi, B. 2003. Tertiary volcanism during extension in the An-

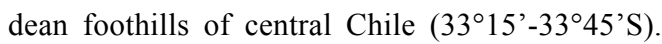
Geological Society of America Bulletin 115 (12): 1523-1537.

Othmann, B.D.; White, W.M.; Patchett, J. 1989. The geochemistry of marine sediments, island arc magma genesis, and crust-mantle recycling. Earth and Planetary Science Letters 94: 1-21.

Pankhurst, R.J.; Weaver, S.D.; Hervé, F.; Larrondo, P. 1999. Mesozoic-Cenozoic evolution of the North Patagonian Batholith in Aysen, southern Chile. Journal of the Geological Society of London 156: 673-694.

Parada, M.A.; Nyström, J.O.; Levi, B. 1999. Multiple source for the Coastal Batholith of central Chile (31$34^{\circ} \mathrm{S}$ ): geochemical and $\mathrm{Sr}-\mathrm{Nd}$ isotopic evidence and tectonic implications. Lithos 46: 505-521.
Pearce, J.A. 1983. Role of sub-continental lithosphere in magma genesis at active continental margins. In Continental Basalts and Mantle Xenoliths (Hawkesworth, C.J.; Norry, M.J.; editors). Shiva Publishing, Nantwich: 203-249. UK.

Pearce, J.A.; Stern, R.J.; Bloomer, S.H.; Fryer, P. 2005. Geochemicalmapping of the Mariana arc-basin system: Implications for the nature and distribution of subduction components. Geochemistry, Geophysics, Geosystems 6, Q07006, doi:10.1029/2004GC000895.

Reich, M.; Parada, M.A.; Palacios, C.; Dietrich, A.; Schultz, F.; Lehmann, B. 2003. Adakite-like signature of Late Miocene intrusions at the Los Pelambres giant porphyry copper deposit in the Andes of central Chile: metallogenic implications. Mineralium Deposita 38: 876-885.

Richards, J.P. 2005. Cumulative factors in the generation of giant calc-alkaline porphyry $\mathrm{Cu}$ deposits. In Super porphyry copper and gold deposits: A global perspective (Porter, T.M.; editor). Porter GeoConsultancy (PGC) Publishing, Linden Park 1: 7-25. South Australia.

Rivano, S.; Godoy, E.; Vergara, M.; Villarroel, R. 1990. Redefinición de la Formación Farellones en la Cordillera de los Andes de Chile Central (32-34 $\left.{ }^{\circ} \mathrm{S}\right)$. Revista Geológica de Chile 17 (2): 205-214.

Shirey, S.; Walker, R. 1995. Carius tube digestion for lowblank rhenium-osmium analysis. Analytical Chemistry 67 (13): 2136-2141.

Skewes, M.A.; Stern, C.R. 1994. Tectonic trigger for the formation of late Miocene $\mathrm{Cu}$-rich megabreccias in the Andes of central Chile. Economic Geology 2: 551-554.

Skewes, M.A.; Stern, C.R. 1996. Late Miocene mineralized breccias in the Andes of central Chile: Sr- and $\mathrm{Nd}$-isotopic evidences for multiple magmatic sources. In Andean copper deposits: new discoveries, mineralization, styles and metallogeny (Camus, F.; Sillitoe, R.; Petersen, R.; editors). Society of Economic Geologists, Special Publication 5: 33-41.

Skewes, M.A.; Stern, C.R.; Holmgren, C.; Contreras, A.; Godoy, S.; Vela, I.; Rivano, S. 1991. Evolución magmática cerca del borde sur del segmento de bajo ángulo de subducción en Chile Central (32-34º S). In Congreso Geológico Chileno, No. 6, Extended Abstracts: 146-148. Viña del Mar.

Sillitoe, R.H. 1974. Tectonic segmentation of the Andes: Implications for magmatism and metallogeny. Nature 250: 542-545.

Stern, C.R. 1991. Role of subduction erosion in the generation of Andean magmas. Geology 19: 78-81. 
Stern, C.R.; Futa, K.; Muehlenbachs, K. 1984. Isotope and trace element data for orogenic andesites from the austral Andes. In Andean Magmatism; chemical and isotopic constraints (Harmon, R.S.; Barreiro, B.A; editors) . Shiva Publication: 31-46. U.K.

Stern, C.; Puig, A. 1991. Geochemical Evolution of Magmatic Rocks in the Vicinity of El Teniente Copper Deposit ( $\left.34^{\circ} \mathrm{S}\right)$, Central Chilean Andes. In Congreso Geológico Chileno, No. 6, Extended Abstracts: 265 267. Viña del Mar.

Stern, C.; Skewes, A. 1995. Miocene to the present magmatic evolution at the northern end of the Andean Southern Volcanic Zone, central Chile. Revista Geológica de Chile 22 (2): 261-272.

Streckeisen, A. 1976. To each plutonic rock its proper name. Earth Science Reviews 12: 1-33.

Sun, S.S.; McDonough, W.F. 1989. Chemical and isotopic systematics of oceanic basalts: implications for mantle composition and process. In Magmatism in ocean basins (Saunders, A.D.; Norry, M.J.; editors). Geological Society of London, Special Publication 42: 313-345.

Thieblemont, D.; Stein, G.; Lescuyer, J.-L. 1997. Gisements epithermeaux et porphyriques: la connexion adakite. C.R. Academie des Sciences Comptes Rendus, serie 2: Sciences de la terre et des planetes, Earth and Planetary Sciences 325: 103-109.

Tollstrup, D.L.; Gill, J.B. 2005. Hafnium systematics of the Mariana arc: Evidence for sediment melt and residual phases. Geological Society of America 33: 737-740.

Vergara, M.; Drake, R. 1978. Edades potasio-argón y su implicancia en la geología regional de Chile Central. Universidad de Chile, Departamento de Geología, Comunicaciones 23: 1-11.

Warnaars, F.W.; Holmgren, C.; Barassi, S. 1985. Porphyry copper and tourmaline breccias at Los Bronces-Río Blanco, Chile. Economic Geology 80: 1544-1565.

Wiedenbeck, M.; Allé, P.; Corfu, F.; Griffin, W.L.; Meier, M.; Oberli, F.; von Quadt, A.; Roddick, J.C.; Spiegel, W. 1995. Three natural zircon standards for $\mathrm{U}-\mathrm{Th}-\mathrm{Pb}$, Lu-Hf, trace element and REE analyses. Geostandard Newsletters 19: 1-23.

Wilson, S.A. 1997. The collection, preparation and testing of USGS reference material BCR-2, Columbia River, Basalt. U.S. Geological Survey Open-File Report 98-00x.

Yáñez, G.A.; Ranero, C.R.; von Huene, R.; Díaz, J. 2001. Magnetic anomaly interpretation across the southern central Andes $\left(32^{\circ}-34^{\circ} \mathrm{S}\right)$ : The role of the Juan Fernández Ridge in the late Tertiary evolution of the margin. Journal of Geophysical Research 106 (B4): 6325-6345.

Zentilli, M.; Pop, N.; Heaman, L.; Boric, R. 2001. Evidence of Proterozoic crust under the Coastal Cordillera of Central Chile: Grenville age xenocrystic zircons in Cretaceous volcanic rocks. In South American Isotope Conference, CD-ROM 642. Chile. 


\section{APPENDIX 1. ANALYTICAL METHODS}

For single-zircon grain LA-ICP-MS U-Pb and Hfisotope analysis, magmatic zircons were separated from $2 \mathrm{~kg}$ samples using routine crushing, grinding. Gemini Table, heavy liquid and Frantz magnetic separation methods in the Geology Department, University of Chile. Preliminary grinding was undertaken in the same facility for samples selected for geochemistry. All geochemical and isotope analyses were carried out in the geochemical facilities at Macquarie University, Sydney, Australia.

For U-Pb geochronology and Hf-isotope analyses, zircon mineral separates were mounted in epoxy in a 2.5 $\mathrm{cm}$ diameter sample-holder and polished. Backscatter electron (BSE)/cathodoluminescence (CL) images were taken of all analysed zircons to study the internal structure of the grains prior to LA-ICP-MS analysis. Mineral ablation was carried out using a LUV213 laser ablation system. LA-ICP-MS analyses were done in an Agilent 4,500 high-sensitivity quadrupole ICPMS providing a sensitivity of more then 200 million counts per second (cps)/ppm for mono-isotopic heavy elements (atomic mass $>85$ ) in standard solution mode. The transporter gas of the ablated material was He. Spot diameters ranged between 50 and $80 \mu \mathrm{m}$, ablation time was about $60 \mathrm{~s}$ and the ablated masses of zircon were approximately 500$1,500 \mathrm{ng}$. Detection limits for heavy elements applying laser ablation sampling are typically $<10 \mathrm{ppb}$ for a $60 \mathrm{~s}$ ablation at $40-50 \mu \mathrm{m}$ resolution. Gas blank ${ }^{208} \mathrm{~Pb}$ signals of 30-40 cps were achieved routinely. The in-house GJ-1 zircon standard (608.5 $\pm 0.4 \mathrm{Ma})$ was used for optimizing ICP-MS operating conditions. Reproducibility and accuracy were controlled by zircon standards $91500(1,065.4 \pm 0.3$ Ma; Wiedenbeck et al.,1995) and Mud Tank (732 $\pm 5 \mathrm{Ma}$; Black and Gulson, 1978). The GLITTER software program for LAM-ICPMS analyses undertakes data reduction and calculation of age estimates from isotopic ratios. Ages are quoted at $2 \sigma$ error intervals.

Hf-isotope analyses were carried out in situ using a Merchantek EO LUV laser-ablation microprobe, attached to a Nu-Plasma multi-collector ICP-MS. Beam diameter of approximately $80 \mu \mathrm{m}$ resulted in total Hf signals of 1 $6 \times 10^{-11}$ A depending on Hf concentrations, which ranged from 0.9 to $1.8 \%$ Hf. Ablation time was $80-120 \mathrm{~s}$ and pit depth 40-60 $\mu \mathrm{m}$; typically a total amount of $50 \mathrm{ng}$ of $\mathrm{Hf}$ was consumed. For correction of the interference of ${ }^{176} \mathrm{Yb}$ on ${ }^{176} \mathrm{Hf}$, a 1 ppm solution of JMC475 Hf spike with 80 ppb $\mathrm{Yb}$ was used with the aim to obtain the appropriate value of ${ }^{176} \mathrm{Yb} /{ }^{172} \mathrm{Yb}$ to calculate ${ }^{176} \mathrm{Yb} /{ }^{177} \mathrm{Hf}$ ratios. The recommended ${ }^{176} \mathrm{Lu} /{ }^{175} \mathrm{Lu}$ ratio of 0.02669 (De Bievre and Taylor, 1993) was used to calculate ${ }^{176} \mathrm{Lu} /{ }^{177} \mathrm{Hf}$ ratios. The 2 SE uncertainty on single analyses of ${ }^{176} \mathrm{Lu} /{ }^{177} \mathrm{Hf}$ ratios was $<1-2 \%$. Typical within run precision (2SE) on the ${ }^{176} \mathrm{Hf} /{ }^{177} \mathrm{Hf}$ ratio analyses were \pm 0.000020 . Hf isotope analyses indicated mean values for ${ }^{176} \mathrm{Hf} /{ }^{177} \mathrm{Hf}$ ratios of JMC475 Hf standard identical to the recommended value of 0.28216 by Nowell et al. (1998). Chondritic values of Blichert-Toft and Albarède (1997) were used for calculation of epsilon Hf values. Depleted-mantle source model ages are based on ${ }^{176} \mathrm{Hf} /{ }^{177} \mathrm{Hf}=0.279718$ and ${ }^{176} \mathrm{Lu} /{ }^{177} \mathrm{Hf}=0.0384$ values which produce ${ }^{176} \mathrm{Hf} /{ }^{177} \mathrm{Hf}=0.28325$ similar to average MORB over $4.56 \mathrm{Ga}$.

Further detailed information on methodology, sample preparation and instrumentation are described by Jackson et al. (2004) for U-Pb analyses and by Griffin et al. (2000) for Hf-isotope measurements.

Major and some trace elements ( $, \mathrm{Cr}, \mathrm{Co}, \mathrm{Ni})$ were analyzed by X-ray fluorescence spectrometry using a Spectro XLAB2000 energy dispersive spectrometer in the Geochemical Analysis Unit, GEMOC, Macquarie University. Samples for major element analysis were prepared as glass discs using a lithium metaborate flux. Trace elements were determined on pressed powder pellets using a PVA binder. Replicate analyses of USGS reference materials (BCR-2, Columbia River Basalt; Wilson, 1997) give precision values of $<1 \mathrm{wt} \%$ relative standard deviation (rsd) for all elements with concentrations $>0.2 \mathrm{wt} \%$, except for $\mathrm{Na}$ which has a precision of $2-3 \mathrm{wt} \%$ rsd. For trace elements typical precision is as follows: $>500 \mathrm{ppm}, 0.5-2.0 \mathrm{wt} \% \mathrm{rsd} ; 100-500 \mathrm{ppm}, 1-3$ wt $\%$ rsd; $10-100$ ppm, 3-10 wt $\%$ rsd; 1-10 ppm, 12-25 wt $\%$ rsd; $<1$ ppm, 25-40 wt $\%$ rsd.

All other trace elements were analyzed by an Agilent 7500cs ICP-MS Octopole Reaction System using standard mode with no collision cell reaction gas. Argon was used as carrier gas. Other instrument parameters include a Pt shield on torch, quartz torch and spray chamber and a self aspirating PFA nebuliser. Standard deviation for replicated measurements on sample FA-3 lies generally between zero and $3 \%$ with one exception for Ba yielding a $7 \%$ standard deviation on its replicate. The standard deviation between the measured and recommended standard values of the analysed (BCR-2) generally ranges from 0 to $4 \%$ with the exception of $\mathrm{Sr}$ and Ba yielding a 12 and $17 \%$, respectively. Instrument drift was corrected by spiking samples and standards with internal standards as ${ }^{6} \mathrm{Li}, \mathrm{As}, \mathrm{Rh}, \mathrm{In}, \mathrm{Tm}$ and Bi. The used digestion procedure employed $100 \mathrm{mg}$ of rock 
powder in $15 \mathrm{ml}$ Teflon Savillex beakers mixed with $\mathrm{HF} / \mathrm{HNO}_{3}$ and $\mathrm{HClO}_{4}$ acids over night. This procedure was repeated several times before finally mixed with $2 \%-\mathrm{HNO}_{3}$ and analysed on the ICP-MS.

For magmatic magnetite analysed $0.2-0.5 \mathrm{~g}$ of handpicked minerals were loaded in Carius tubes (Shirley and Walker, 1995). Re and Os spikes and $16 \mathrm{ml}$ of Aqua Regia were added and kept under cold conditions to avoid evaporation and early chemical reaction, then sealed. Afterwards, the tube was placed into an oven at $240^{\circ} \mathrm{C}$ overnight. Os was separated from the solution in a two-stage distillation process (Nagler and Frei, 1997), then purified and loaded with $\mathrm{Ba}(\mathrm{OH})_{2}$ on a Ptfilament for analysis by a negative thermal ion mass spectrometer (NTIMS-VG54 mass spectrometer; Creaser et al., 1997). Re was separated after Os by using the remaining acid solution, After drying the solution, it was re-dissolved in $0.1 \mathrm{HNO}_{3}$. Re was extracted and purified by using AG1-X8 (100-200 mesh) resins. After- wards Re was loaded with $\mathrm{Ba}(\mathrm{SO})_{4}$ on Pt-filament and analysed by NTIMS.

Whole rock isotopic analyses were obtained by using the multi-collector Nu Plasma Instrument ICP-MS. The chemical separation for $\mathrm{Sr}-\mathrm{Nd}$ isotopes from the whole rock samples was obtained by using cation and anion exchange resin columns. An exponential correction for mass fractionation was applied using ${ }^{146} \mathrm{Nd} /{ }^{145} \mathrm{Nd}=2.07204$ to avoid an iterative correction for the interference of ${ }^{144} \mathrm{Sm}$ on ${ }^{144} \mathrm{Nd}$. The value for ${ }^{146} \mathrm{Nd} /{ }^{145} \mathrm{Nd}$ is an average of results obtained on a $1 \mathrm{ppm}$ JMC321 Nd solution and is normalised to ${ }^{146} \mathrm{Nd} /{ }^{144} \mathrm{Nd}=0.7219$. The interference of ${ }^{144} \mathrm{Sm}$ on ${ }^{144} \mathrm{Nd}$ was corrected by measuring ${ }^{147} \mathrm{Sm}$ and using ${ }^{144} \mathrm{Sm} /{ }^{147} \mathrm{Sm}=0.2070$. Corrections for mass fractionation of $\mathrm{Sr}$ and $\mathrm{Rb}$ isotope ratios are made using an exponential law using a normalizing value for ${ }^{86} \mathrm{Sr} /{ }^{88} \mathrm{Sr}=0.1194$. The precision $(2 \mathrm{sd})$ and accuracy of standard analyses generally average for $\mathrm{Sr}$ isotpes 0.000066 and for $\mathrm{Nd}$ $0.000040 \mathrm{a}$. 